\title{
On the Brill-Noether theory for K3 surfaces
}

\author{
Maxim Leyenson
}

\begin{abstract}
Let $(S, H)$ be a polarized K3 surface. We define Brill-Noether filtration on moduli spaces of vector bundles on $S$. Assume that $\left(c_{1}(E), H\right)>0$ for a sheaf $E$ in the moduli space. We give a formula for the expected dimension of the Brill-Noether subschemes. Following the classical theory for curves, we give a notion of Brill-Noether generic K3 surfaces.

Studying correspondences between moduli spaces of coherent sheaves of different ranks on $S$, we prove our main theorem: polarized K3 surface which is generic in sense of moduli is also generic in sense of Brill-Noether theory (here $H$ is the positive generator of the Picard group of $S$ ). In case of algebraic curves such a theorem, proved by Griffiths and Harris and, independently, by Lazarsfeld, is sometimes called "the strong theorem of the Brill-Noether theory".

We finish by considering a number of projective examples. In particular, we construct explicitly Brill-Noether special K3 surfaces of genus 5 and 6 and show the relation with the theory of Brill-Noether special curves.
\end{abstract}

\section{Contents}

$1 \quad$ Introduction . . . . . . . . . . . . . . . . 2

1.1 Classical Brill-Noether theorv. . . . . . . . . . . . . . 2

1.2 Generalizations. ............... 3

1.3 The case of $\mathrm{K} 3$ surfaces. . . . . . . . . . . . . . . . . . . 4

1.4 Techniques................. 5

1.5 Structure of the paper. . . . . . . . . . . . . . . . . 6

1.6 Acknowledgments. . . . . . . . . . . . . 6

2 Preliminaries: moduli spaces of acceptable sheaves on a K3 surface. 6

3 Brill-Noether stratification of $M_{H}(v)$ and statement of the main theorem

4 The correspondence $\mathcal{A}_{r r^{\prime}}$. . . . . . . . . . . . . . . . . . . . . . 12

5 A few technical lemmas ................. 14

6 Numerical structure of the correspondence $\mathcal{A}$ on a K3 surface . . 16

7 Four special cases. . . . . . . . . . . . . . . 17

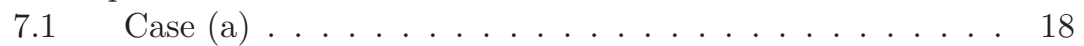

7.2 Case (b) and proof of the main theorem . . . . . . . . . . 20

7.3 Case (c) . . . . . . . . . . . . . . . 22

7.4 Case (d) . . . . . . . . . . . . . . . . 24 
$8 \quad$ Examples . . . . . . . . . . . . . . . . . . . . 25

$8.1 \quad$ Example: $q=3, d=3 \ldots \ldots \ldots \ldots$

$8.2 \quad$ Example: $q=4 . d=5 \ldots \ldots \ldots \ldots . \ldots \ldots$

$8.3 \quad$ Geometrv of $W_{1}^{1}(C)$ for a trigonal curve of genus five . . 27

8.4 Example of a Brill-Noether special K3 of genus 5 . . . . 29

$8.5 \quad$ Example: $a=5 . d=5 \ldots \ldots \ldots \ldots \ldots$

$8.6 \quad$ Example: $a=5 . d=4 \ldots \ldots \ldots \ldots \ldots$

8.7 Example of a Brill-Noether special K3 of genus 6 $\ldots . .34$

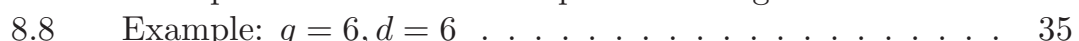

\section{Introduction}

\subsection{Classical Brill-Noether theory.}

We start by recalling some results of classical Brill-Noether theory. Let $C$ be a smooth projective curve of genus $g$ over an algebraically closed field $k$ of characteristics 0 ; consider the Picard variety $\operatorname{Pic}^{d}(C)$ parameterizing degree $d$ line bundles on $C$. For a line bundle $L$ on $C$ one can define cohomology groups with coefficients in $L, H^{0}(C, L)$ and $H^{1}(C, L)$; these are vector spaces over the base field $k$. The vector space $H^{0}(C, L)$ can be interpreted as the space of global sections of $L$. Let us denote by $h^{i}(C, L)$ the dimension of the vector space $H^{i}(C, L)$ over $k, i=0,1$. These numbers are related by the RiemannRoch formula

$$
h^{0}(C, L)-h^{1}(C, L)=\chi(L)=d+1-g,
$$

thus, given $h^{0}(C, L)$, we can compute $h^{1}(C, L)$ from the formula above.

Therefore, the Picard variety $\operatorname{Pic}^{d}(C)$ has a natural filtration given by the value of $h^{0}(C, L)$,

$$
\operatorname{Pic}^{d}(C) \supset W_{d}^{0}(C) \supset W_{d}^{1}(C) \supset \ldots,
$$

where $W_{d}^{r}(C)$ is the set of isomorphism classes of the degree $d$ line bundles $L$ which satisfy the condition $h^{0}(L) \geq r+1$. (The shift by +1 appears for historical reasons: if $h^{0}(C, L)=r+1$, then $r$ is the dimension of the linear system $\left.|L|\right)$.

One can prove that each $W_{d}^{r}(C)$ is a set of points of a closed subvariety in the Picard variety $\operatorname{Pic}^{d}(C)$, and it is classically known that many questions of the projective geometry of $C$ can be reformulated in terms of the geometry of these subvarieties, named "the Brill-Noether loci" after Brill and Noether who did the first essential study of them in [1 in 1873. (The notation $W_{d}^{r}$ itself goes back to Brill and Noether.) For example, the variety $W_{2}^{1}(C)$ is not empty iff the curve $C$ is hyperelliptic, and if $C$ can be represented as a plane curve of degree $d$, then $W_{d}^{2}(C)$ is not empty.

What is known about the geometry of the Brill-Noether loci for a curve $C$ ? We formulate only a few results. First, one can construct two vector bundles, $E$ and $F$, on a Picard variety $\operatorname{Pic}^{d}(C)$, and a map $\sigma: E \rightarrow F$ in such a way that $W_{d}^{r}$ is equal to the degeneration subvariety of $D_{k}(\sigma)$ for a certain $k$, where

$$
D_{k}(\sigma)=\left\{x \in \operatorname{Pic}^{d}(C): \operatorname{rank} \sigma(x) \leq k\right\}
$$


(see, e.g., 13, Chapter 14.) In such a situation one can define the "expected" (or "virtual") dimension of the variety $W_{d}^{r}$ in such a way that if the map $\sigma$ is "generic" in an appropriate sense, then the dimension of the degeneration variety $W_{d}^{r}$ is equal to its expected dimension. The expected dimension of $W_{d}^{r}(C)$ depends only on the numbers $g, d$ and $r$, but not on the curve $C$ itself, and is classically denoted as $\rho(r, g, d)$. One has

$$
\rho(r, g, d)=g-(r+1)(g-d+r)
$$

and $\rho(r, g, d)$ is called "the Brill-Noether number".

If, for a given curve $C$ and numbers $r$ and $d$, the variety $W_{d}^{r}(C)$ has the "expected" dimension $\rho(r, g, d)$, then much is known about the geometry of $W_{d}^{r}(C)$, for example, one can compute its cohomology class (in the case $k=\mathbb{C}$ ) in the cohomology ring of the Picard variety (A. Poincare), its normal invariants, and so on. (See [6] for many more details).

The curve $C$ is said to be "Brill-Noether general" if

(a) whenever $\rho(r, g, d)<0$ (the expected dimension of $W_{d}^{r}(C)$ is negative), the variety $W_{d}^{r}(C)$ is empty, and

(b) whenever $\rho(r, g, d) \geq 0$, the variety $W_{d}^{r}(C)$ is not empty and $\operatorname{dim} W_{d}^{r}(C)=$ $\rho(r, g, d)$ (i.e., $W_{d}^{r}(C)$ is of expected dimension).

It was conjectured by Brill and Noether that a generic curve $C$ in the moduli space of curves of genus $g$ is (what we call now) Brill-Noether general. The first proof was given by Griffiths and Harris in 1980 ( $[\underline{5}$ ) and used a degeneration argument. It was also observed (Miles Reid, Tyurin, Donagi-Morrison, Lazarsfeld) that if the curve $C$ can be realized as a hyperplane section of some K3 surface $S$, then the geometry of the Brill-Noether loci $W_{d}^{r}(C)$ is closely related to the geometry of the surface $S$. Lazarsfeld proved that if $C$ is a curve which can be embedded into a $\mathrm{K} 3$ surface $S$ in such a way that the linear system $|C|$ does not contain non-reduced or reducible curves, then whenever $\rho(r, g, d)<0$ the variety $W_{d}^{r}(C)$ is empty.

\subsection{Generalizations.}

There are two most natural ways to generalize the classical Brill-Noether theory: first, instead of studying line bundles one can consider coherent sheaves of arbitrary rank $r$. (Unfortunately, the dimension of the linear system $L$, as $r$ in the notation $W_{d}^{r}$, and the rank of a vector bundle are classically denoted by the same letter $r$.) Second, instead of studying curves, one can consider varieties $X$ of dimension greater than one. One can go in both directions simultaneously, fixing a variety $X$ equipped with an ample divisor class $H$ and considering the moduli space $M=M_{H}(r, c)$ of $H$ - stable vector bundles (or coherent sheaves) of rank $r$ and Chern class $c$, and studying the subschemes in $M$ defined by the conditions $\left\{h^{i}(X, E) \geq k_{i}\right\}$.

Going in the first direction, Newstead and others studied the Brill-Noether loci in the moduli spaces of stable rank $r$ vector bundles on curves (their results 
are published in a sequence of papers in 1994-2004). Mumford's study of special 0-cycles, done in the Chapters 19-21 of 4] in order to construct the Picard scheme of an algebraic surface $S$, can be interpreted as a proof of non-emptiness of certain Brill-Noether varieties in the moduli space of rank 1 torsion free sheaves on $S$. Goettsche and Hirschowitz studied the Brill-Noether loci in the moduli spaces of stable vector bundles on the projective plane $\mathbb{P}^{2}$. It is known that if $S$ is an algebraic surface over $k=\mathbb{C}$, then the geometry of the BrillNoether loci in the moduli spaces of vector bundles on $S$ is connected with the invariants of smooth structure of $S$.(For example, Ellingsrud, Stromme and Le Potier studied the Brill-Noether stratification in the moduli space of stable vector bundles on $\mathbb{P}^{2}$ in order to compute some Donaldson invariants of $\mathbb{P}^{2}$ ).

\subsection{The case of K3 surfaces.}

We consider the Brill-Noether filtration in the moduli space of stable vector bundles on K3 surfaces.

In general, a vector bundle $E$ on an algebraic surface $S$ has three Betti numbers, $h^{0}(S, E), h^{1}(S, E)$, and $h^{2}(S, E)$, which are related by the RiemannRoch theorem

$$
h^{0}(S, E)-h^{1}(S, E)+h^{2}(S, E)=\chi(S, E),
$$

and therefore one naturally has to consider a bifiltration in the moduli spaces of stable vector bundles on $S$ given by the condition $h^{0}(S, E) \geq k, h^{1}(S, E) \geq l$. However, if $S$ is a K3 surface, one can prove that the second cohomology group $H^{2}(S, E)$ vanish for all stable vector bundles $E$ such that $\left(c_{1}(E), H\right)>0$. It follows that one has to consider only two Betti numbers which are subject to one relation, and therefore the situation in the case of K3 is simpler and it is enough to study the filtration defined by the dimension of the spaces of global sections.

Let $S$ be a $\mathrm{K} 3$ surface. Let $a \in \operatorname{Pic}(S), d \in \mathbb{Z}$, and let $M=M_{H}(r, a, d)$ be the moduli space of $H$-stable vector bundles $E$ on $S$ which satisfy $\operatorname{rk}(E)=r$, $c_{1}(E)=a$ and $c_{2}(E)=d$. We define the Brill-Noether filtration on $M$ as

$$
B N_{k}(M) \stackrel{\text { def }}{=} B N_{k}(r, a, d)(S) \stackrel{\text { def }}{=}\left\{[E] \in M_{H}(r, a, d): h^{0}(S, E) \geq k\right\} .
$$

We realize $B N_{k}(M)$ as the degeneration variety of a morphism of vector bundles $\sigma: E \rightarrow F$ on the moduli space $M$. We then compute the expected dimension of the Brill-Noether loci $B N_{k}(M)$, which we denote as v.dim $B N_{k}(M)$ ("v" stands for "virtual").

It is natural to ask, then, whether, at least for a "general" K3 surface $S$, the numeric condition v.dim $B N_{k}(M)<0$ implies that the variety $B N_{k}(M)$ is empty, and whether v.dim $B N_{k}(M) \geq 0$ implies that $B N_{k}(M)$ is not empty and is of the expected dimension.

We give a partial answer to this question. To summarize our results shortly,

(1) we give examples of situations when the expected dimension of the BrillNoether subscheme $B N_{k}(v)$ is greater than zero, but the corresponding 
subscheme is empty, in contrast with the classical case, and define a "good" geographic region in the space of numerical parameters $(g, d, r, k)$ where we expect the Brill-Noether filtration to behave in the expected way for a generic K3 surface;

(2) we prove that if $S$ is a K3 surface with Pic $S \simeq \mathbb{Z} a$, where $a$ is the positive generator of Pic $S$, and the generic curve in the linear system $|a|$ is BrillNoether general in the classical sense, then the Brill-Noether filtration on the moduli space $M_{H}(r, a, d)$ has the expected behavior in the good geographic region, and consists of empty varieties outside of the good region, i.e., in this case we have a full answer to the question we study. A theorem of Lazarsfeld implies that the second condition above is extra, i.e., that for a K3-surface $S$ with $\operatorname{Pic} S \simeq \mathbb{Z} a$, where $a$ is the positive generator of Pic $S$, the generic curve $C$ in the linear system $|a|$ is BrillNoether general. Thus a generic polarized K3 surface is Brill-Noether general in the "good" region.

We also give some examples of Brill-Noether-special K3 surfaces (with rank of Pic $S>1$ ), and give some applications to the birational geometry of the moduli spaces of vector bundles on K3 surfaces.

\subsection{Techniques.}

Below I summarize the techniques we use.

First, we extend the problem to include certain coherent sheaves of rank 0 and 1 , thus extending our picture to the moduli spaces of all the ranks $r \geq 0$. For rank 0, studying the corresponding Brill-Noether filtration on the moduli space $M(0, a, d)$ is equivalent to studying the classical Brill-Noether theory for curves on $S$ in the linear system $|a|$, and for sheaves of rank 1 studying of the Brill-Noether filtration on $M(1, a, d)$ is equivalent to the study of the 0-cycles on $S$ which fail to impose independent conditions on curves in the linear system $|a|$.

Then, continuing the line of thought of Tyurin in 14, we define some system of correspondences which relate Brill-Noether varieties in the moduli spaces of sheaves of various ranks. We can use this system of correspondences to obtain information about the geometry of the Brill-Noether variety $B N_{k}(r, a, d)$ for a given $k$ and $r$, provided that we can guarantee that for a certain $r^{\prime}$, depending on $r, k, a$ and $d$,

(1) for a generic vector bundle $[E] \in M_{H}\left(r^{\prime}, a, d\right)$ one has $h^{1}(S, E)=0$, and

(2) generic vector bundle in $M_{H}\left(r^{\prime}, a, d\right)$ is globally generated.

However, this seem to be a hard question on its own. In our study of the Brill-Noether theory on $S$ we apply the correspondence twice, the first time to translate some information about the special linear systems on curves on the surface $S$ into information about the behavior of the generic vector bundles $[E] \in$ $M_{H}\left(r^{\prime}, a, d\right)$ for various ranks $r^{\prime}$, thus reversing the line of though of Lazarsfeld 
and answering, under certain conditions, the question about the behavior of the generic vector bundles posed above, and the second time to translate the information about the generic vector bundles in the moduli spaces $M_{H}\left(r^{\prime}, a, d\right)$ into the information about the given Brill-Noether variety $B N_{k}(r, a, d)$.

\subsection{Structure of the paper.}

The proof of some technical results will appear in a separate publication [22]. The proof of the main theorem is given completely in this paper.

\subsection{Acknowledgments.}

My interest in the Brill-Noether theory was prompted by a conversation with prof. M.S. Narasimhan at the International Center for Theoretical physics (ICTP), Trieste, Italy in 1999. I would like to thank prof. M.S. Narasimhan and ICTP for hospitality.

The proof of the main theorem is based on the ideas of Robert Lazarsfeld and Andrey Tyurin. This paper is a result of my attempt to understand the "Cycles, curves and vector bundles on algebraic surfaces" by Andrey Tyurin (14).

I am thankful do Dima Arinkin for reading the preliminary version of the manuscript.

I am especially thankful to Andrey Levin of the Institute for Theoretical and Experimental Physics (ITEP, Moscow) for numerous discussions, help, encouragement and psychological support.

The proof of the technical results mentioned in will hopefully appear in the second part of the paper.

\section{Preliminaries: moduli spaces of acceptable sheaves on a K3 surface.}

Let $S$ be a K3 surface over an algebraically closed field $k$ of characteristic 0 . Let $K(S)=K^{0}(S), K^{\prime}(S)=\mathbb{Z} \oplus \operatorname{Num} S \oplus \mathbb{Z}$, and $K^{\prime \prime}=\mathbb{Z}^{3}$. Consider the maps

$$
K(S) \stackrel{\text { ch }}{\rightarrow} K^{\prime}(S) \stackrel{\epsilon}{\rightarrow} K^{\prime \prime}
$$

where the Chern character map ch takes a class of a locally free sheaf $E$ to its Chern character $\operatorname{ch}(E)=\left(\operatorname{rk} E, \operatorname{ch}_{1}(E), \operatorname{ch}_{2}(E)\right)$, and $\epsilon\left(r, v_{1}, v_{2}\right)=(r, g, d)$, where $g=\frac{v_{1}^{2}+2}{2}$, and $d=\frac{v_{1}^{2}-2 v_{2}}{2}$. Note that $\operatorname{ch}_{2}(E)=\left(c_{1}(E)^{2}-2 c_{2}(E)\right) / 2 \in \mathbb{Z}$, since Num $S$ is an even lattice, so the map ch is correctly defined. Note also that for a locally free sheaf $E$ one has $\epsilon(v([E]))=(\operatorname{rk} E, g, d)$, where $g$ is the arithmetic genus of a curve in the linear system $\left|c_{1}(E)\right|$ in the case it is not empty, and $d=c_{2}(E)$. Note that linear equivalence on $S$ coincides with numerical equivalence, since $H^{1}\left(S, \mathcal{O}_{S}\right)=0$. 
If $a \in \operatorname{Num} S$, we denote by $|a|$ the linear system $\mathbb{P} H^{0}\left(S, L_{a}\right)^{\vee}$, where $L_{a}$ is the unique invertible sheaf on $S$ with $c_{1}\left(L_{a}\right)=a$, and by $|a|^{s}$ the open subscheme in $|a|$ parametrizing smooth irreducible curves.

We say that a coherent sheaf $F$ on $S$ is acceptable ${ }^{1}$ if it satisfies one of the following conditions:

1. rk $F \geq 2$ and $F$ is locally free;

2. $\operatorname{rk} F=1$ and $F$ is torsion free;

3. rk $F=0$ and $F$ is isomorphic to the direct image of an invertible sheaf on a smooth irreducible curve on $S$.

We give some justification to this definition later.

Let $H \in N u m(S)$ be a class of an ample divisor, let $v=\left(r, v_{1}, v_{2}\right) \in K^{\prime}(S)$ such that $\left(v_{1}, H\right)>0$, and let $M(v)=M_{H}(v)$ be the moduli space of $H$-stable acceptable coherent sheaves on $S$ with Chern character $v$. (This moduli space can be constructed as an open subscheme in the moduli space of stable coherent sheaves. For the construction of the latter see, for example, [17.)

Let $\epsilon(v)=(r, g, d)$. We define

$$
\beta=v_{2}=g-1-d
$$

and

$$
\alpha=-\beta=d+1-g
$$

\subsection{Definition.}

$$
\rho(r, g, d):=g-(r+1)(r+g-d)
$$

Note that if $d<0$, then $\rho(r, g, d)<0$.

For any $r \in \mathbb{Z}$ we let $\bar{r}=r-1$.

2.2 Theorem (Shigeru Mukai, [7, 8]). If $r \geq 2$ and $\rho(\bar{r}, g, d) \geq 0$, then $M_{H}(v)$ is a smooth scheme of dimension $2 \rho(\bar{r}, g, d)=2(g-r(r-\alpha))$. If $r \geq 2$ and $\rho(\bar{r}, g, d)<0$, then $M_{H}(v)$ is empty. ${ }^{2}$

2.3 Lemma (case $r=1$ ). There is an isomorphism

$$
i_{1}: \operatorname{Hilb}^{d}(S) \stackrel{\sim}{\rightarrow} M_{H}(1, a, \beta),
$$

which takes a 0 -dimensional subscheme $\xi \subset S$ of length $d$ to the isomorphism class $\left[J_{\xi}\left(L_{a}\right)\right]$, where $L_{a}$ is the unique invertible sheaf on $S$ with Chern class $c_{1}\left(L_{a}\right)=a$.

It follows that $M_{H}(1, a, \beta)$ is a smooth irreducible variety of dimension $2 d$ if $d \geq 0$, and is empty if $d<0$.

\footnotetext{
${ }^{1}$ This is not a standard terminology

${ }^{2}$ Yoshioka proved that if $\rho \geq 0$, then $M(v)$ is not empty and is irreducible. We are not using his result.
} 
2.4 Lemma (case $r=0$ ). There is an isomorphism

$$
i_{0}: \mathrm{Pic}^{2 g-2-d}\left(|a|^{s}\right) \stackrel{\sim}{\rightarrow} M_{H}(0, a, \beta),
$$

where $\operatorname{Pic}^{2 g-2-d}\left(|a|^{s}\right)$ is the "Picard variety of degree $2 g-2-d$ line bundles on curves in the linear system $|a|^{s "}$. A point in $\operatorname{Pic}^{2 g-2-d}\left(|a|^{s}\right)$ is a pair $(C, B)$, where $C$ is a smooth irreducible curve in $|a|$ and $B \in \mathrm{Pic}^{2 g-2-d}(C)$. In the notations of [2], $\mathrm{Pic}^{2 g-2-d}\left(|a|^{s}\right)$ is a component of the relative Picard variety $\underline{\operatorname{Pic}}_{\mathcal{C} /|a|^{s}}$, where $\mathcal{C} /|a|^{s}$ is the universal curve over $|a|^{s}$. The isomorphism $i_{0}$ takes a pair $(C, B)$ to the direct image $\left(i_{C}\right)_{*}(B)$, where $i_{C}: C \rightarrow B$ is the canonical embedding.

In particular, $M(0, a, \beta)$ is an irreducible variety of dimention $2 g$.

2.5 Remark. The formula $\operatorname{dim} M(r, a, \beta)=2 \rho(\bar{r}, g, d)$ remains valid for $r=0$ and $r=1$.

\section{Brill-Noether stratification of $M_{H}(v)$ and statement of the main theorem}

3.1 Lemma: For every $[F] \in M_{H}(v)$ we have $H^{2}(S, F)=0$. (Recall that $\left(v_{1}, H\right)>0$.)

This lemma prompts the following definition:

\subsection{Definition:}

$$
B N_{k}(v)=\left\{[F]: \operatorname{ch}(F)=v, F \text { is acceptable and } H \text {-stable, } h^{0}(S, F) \geq k\right\}
$$

3.3 Remark (case $r=0$ ). If $r=0$, we have

$$
B N_{k}(0, a, \beta)=W_{2 g-2-d}^{\bar{k}}\left(|a|^{s}\right),
$$

where $W_{d}^{r}\left(|a|^{s}\right)$ is the relative Brill-Noether scheme over the linear system $|a|^{s}$. Note that there is a morphism $W_{d}^{r}\left(|a|^{s}\right) \rightarrow|a|^{s}$ with fibers $W_{d}^{r}(C)$.

3.4 Special 0-subschemes on a surface. Let $S$ be an algebraic surface, $L$ be an invertible sheaf on $S$, and $\xi \in \operatorname{Hilb}^{d}(S)$ be 0 -subscheme in $S$. The index of speciality of $\xi$ with respect to $L$, denoted as $\delta(\xi, L)$, is defined by the equality

$$
h^{0}\left(S, J_{\xi}(L)\right)=h^{0}(S, L)-d+\delta(\xi, L)
$$

It follows that $\delta(\xi, L) \geq 0$, and $\delta(\xi, L)>0$ if and only if $\xi$ fails to impose $d$ independent conditions on curves in the linear system $|L|$. If $\delta(\xi, L)>0, \xi$ is said to be special with respect to $L$. Considering the adjunction sequence

$$
0 \rightarrow J_{\xi}(L) \rightarrow L \rightarrow \mathcal{O}_{\xi}(L) \rightarrow 0,
$$

one can see that $\delta(\xi, L)=h^{1}\left(S, J_{\xi}(L)\right)-h^{1}(S, L)$.

For a given $\delta \geq 0$ the set of 0 -subschemes on $S$ which satisfy $\delta(\xi, L) \geq \delta$ form a closed subscheme in the Hilbert scheme of points $\operatorname{Hilb}^{d}(S)$ which we denote as $\operatorname{Hilb}_{(L, \delta)}^{d}(S)$. 
3.5 Special $\mathbf{0}$-subschemes on a K3 surface. If $S$ is a K3 surface and $L$ is ample, we have $\delta(\xi, L)=h^{1}\left(J_{\xi}(L)\right)=h^{0}\left(S, J_{\xi}(L)\right)-\chi\left(S, J_{\xi}(L)\right)=h^{0}\left(S, J_{\xi}(L)\right)+$ $\alpha-2$.

Corollary: case $r=1$. On a K3 surface $S$ there is an equality

$$
B N_{k}(1, a, \beta)=\operatorname{Hilb}_{(L, k+\alpha-2)}^{d}(S) .
$$

\subsection{Simple Caley-Bacharash 0-cycles.}

Definition. A 0-subscheme $\xi \subset S$ of length $d$ is called simple if it can be written as a sum of $d$ distinct points on $S, \xi=\left[p_{1}\right]+\cdots+\left[p_{d}\right], p_{i} \neq p_{j}$.

Definition. If $L$ is an invertible sheaf on $S$ and $\xi$ is a simple 0 -cycle on $S$, then $\xi$ is said to be Caley-Bacharash with respect to $L$ if for every point $p_{i} \in \xi$ and every curve $C$ in the linear system $|L|$ such that all the points $p_{i}, i \neq d$, belong to $C$ we also have $p_{i} \in C$, (i.e., if $h^{0}\left(S, J_{\xi-p_{i}}(L)\right)=h^{0}\left(S, J_{\xi}(L)\right)$ for every $i$.)

For example, if $C$ and $D$ are plane curves and $\xi=C \cap D$ is a simple 0cycle, then $\xi$ is Caley-Bacharash with respect to the linear system $\mid \mathcal{O}_{\mathbb{P}^{2}}(\operatorname{deg} C+$ $\operatorname{deg} D-3) \mid$. In particular, every plane cubic containing eight out of nine points of intersection of two fixed plane cubics also contains the ninth point (CaleyBacharash theorem.)

Let $\xi=p_{1}+\cdots+p_{d}$ be a simple Caley-Bacharash 0-cycle on $S$. One can see that $\delta(\xi, L)=\delta\left(\xi-p_{i}, L\right)+1$ for each $i$. It follows that $\xi$ is special with respect to $L$. One can prove that for each given $\delta>0$ simple Caley-Bacharash 0 -cycles form an open subscheme in $\operatorname{Hilb}_{(L, \delta)}^{d}(S)$.

We let $\operatorname{Hilb}_{(L, \delta)^{\circ}}^{d}(S)=\operatorname{Hilb}_{(L, \delta)}^{d}(S)-\operatorname{Hilb}_{(L, \delta+1)}^{d}(S)$.

3.7 Lemma. Let us fix $v$, and assume that $M(v)$ is not empty. Let us substitute $M(v)$ with any of its irreducible components ${ }^{3}$.

1. One can construct vector bundles $E$ and $F$ on $M(v)$ and a map $\sigma: E \rightarrow F$ in such a way that $B N_{k}(v)$ is the set of points $x \in M(v)$ at which the rank of $\sigma$ drops by a certain number $l$. In particular, $B N_{k}(v)$ is the set of points of a closed subscheme $D_{l}(\sigma)$ in the moduli space $M(v)$. (Abusing notations, we sometimes denote this subscheme as $\left.B N_{k}(v)\right)$.

2. If $B N_{k}(v)$ is not empty, then

$$
\operatorname{codim}_{M(v)} B N_{k}(M(v)) \leq k(k-\chi(v)),
$$

where $\chi(v)=\chi(S, F)$ for $[F] \in M(v) .{ }^{4}$

3.8 Definition. We define the expected, or virtual, dimension of $B N_{k}(M(v))$ by

$$
\begin{aligned}
\mathrm{v} \cdot \operatorname{dim} B N_{k}(M(v)) & =\operatorname{dim} M(v)-k(k-\chi(v)) \\
& =2 \rho(\bar{r}, g, d)-k(k-\chi)
\end{aligned}
$$

\footnotetext{
${ }^{3}$ We do not use the strong result that $M(v)$ is irreducible

${ }^{4}$ Note that the same formula is true in the classical Brill-Noether theory for line bundles on curves, see, e.g., [16].
} 
where $\chi=\chi(v)=2 r+\beta$ is the Euler characteristics of any $F \in M_{H}(v)$.

Let us fix $S, H, a \in \operatorname{Num} S$ and $\beta \in \mathbb{Z}$. Let $r_{0}$ be the positive root of the equation

$$
\rho\left(\bar{r}_{0}, g, d\right)=g-r_{0}\left(r_{0}+\beta\right)=0
$$

Note that the moduli space $M_{H}(r, a, \beta)$ is empty if $r>r_{0}$. Let $k_{0}=2 r_{0}+\beta$ and $k_{1}=r_{0}+\beta$.

We define the following domains in the plane with coordinates $(k, r)$ :

$$
\begin{aligned}
& D_{0}=\{(k, r): k \geq 0, k \geq 2 r+\beta, k<r\}, \\
& D_{1}=\left\{(k, r): r \geq 0, k \geq 2 r+\beta, r-k \geq r_{0}-k_{0}\right\}, \\
& D_{2}=\left\{(k, r): r \geq 0, r-k<r_{0}-k_{0}, \operatorname{v} \cdot \operatorname{dim} B N_{k}(r, a, \beta) \geq 0\right\}, \\
& D_{3}=\left\{(k, r): r \geq 0, k \geq 2 r+\beta, v \cdot \operatorname{dim} B N_{k}(r, a, \beta)<0\right\},
\end{aligned}
$$

as one the Figure 1]

Note that the domain $D_{0}$ is empty if $\beta<0$, and

$$
D_{0} \cup D_{1} \cup D_{2}=\left\{(k, r): k \geq \chi(v)=2 r+\beta, \operatorname{v} \cdot \operatorname{dim} B N_{k}(r, a, \beta) \geq 0\right\}
$$

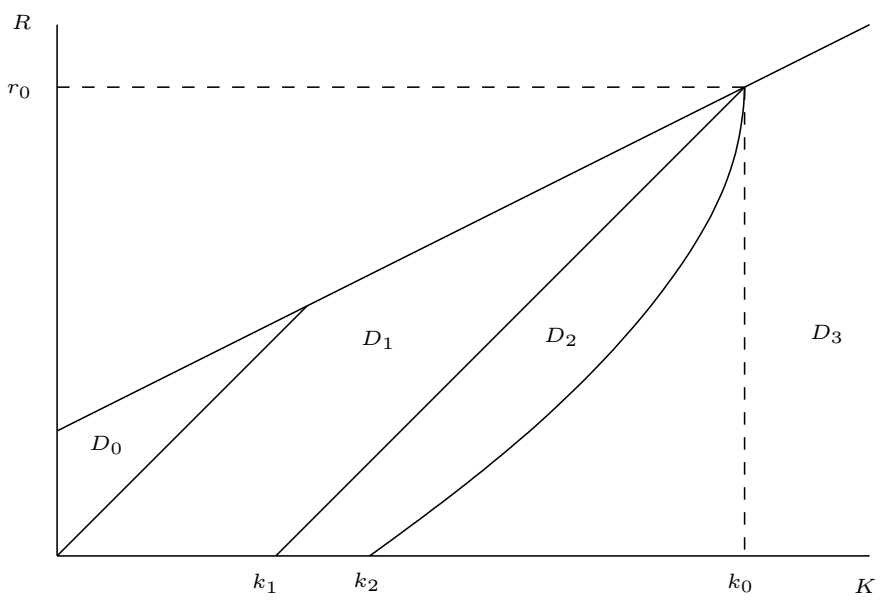

Figure 1: Geography in the $(k, r)$-plane, case $\beta<0$.

Let

$$
V_{d}^{\bar{r}}\left(|a|^{s}\right)=W_{d}^{\bar{r}}\left(|a|^{s}\right)-W_{d}^{\overline{r+1}}\left(|a|^{s}\right)
$$

be the subscheme in the relative Picard variety $\operatorname{Pic}^{d}\left(|a|^{s}\right)$ parametrizing pairs $(C, B)$ for which $h^{0}(C, B)=r+1$.

Let $\left(V_{d}^{\bar{r}}\right)^{\prime}\left(|a|^{s}\right)$ be the open subscheme in $V_{d}^{\bar{r}}\left(|a|^{s}\right)$ parametrizing pairs $(C, B)$ for which $K_{C} B^{-1}$ is globally generated, and let $\left(V_{d}^{\bar{r}}\right)^{\prime \prime}\left(|a|^{s}\right)$ be the open subscheme in $V_{d}^{\bar{r}}\left(|a|^{s}\right)$ parametrizing pairs $(C, B)$ for which both $B$ and $K_{C} B^{-1}$ are globally generated. 
It is known (the "existence theorem" of the classical Brill-Noether theory; Kempf, Kleiman-Laksov, Fulton-Lazarsfeld) that for a smooth genus $g$ curve $C$ the scheme $V_{d}^{r}(C)$ is not empty whenever $\rho(\bar{r}, d, g) \geq 0$. However, there are situations when $\rho(\bar{r}, d, g) \geq 0$, but $\left(V_{d}^{r}(C)\right)^{\prime \prime}$ is empty; the minimal genus for which this occurs is $g=5$. For example, if $C$ is a smooth plane quintic, the variety $V_{5}^{1}(C)$ is two-dimensional, but $\left(V_{5}^{1}\right)^{\prime \prime}(C)$ is empty; some other examples are studied in the section 8

Let us fix a polarized genus $g \mathrm{~K} 3$ surface $(S, a)$ and integers $r \geq 0$ and $d \geq 0$ such that $\rho(\bar{r}, g, d) \geq 0$. Consider the following three conditions:

$C_{1}$ : The scheme $\left(V_{d}^{\bar{r}}\right)^{\prime \prime}\left(|a|^{s}\right)$ is not empty;

$C_{2}$ : The linear system $|a|^{s}$ contains a Brill-Noether general curve;

$C_{3}:$ Pic $S=\mathbb{Z} a$

Note that the condition $C_{3}$ and results of Lazarsfeld imply the condition $C_{2}$, and the condition $C_{2}$ implies the condition $C_{1}$.

3.9 Main Theorem. Let $v=(r, a, \beta) \in K^{\prime}(S)$ and $\epsilon(v)=(r, g, d)$, and assume that $M(r, a, \beta)$ is not empty. Assume further that either $\operatorname{Pic}(S)=\mathbb{Z} a$, or $(a, H)=1$. Then

0 . If $(k, r) \in D_{0}$, then either $B N_{k}(r, a, \beta)$ is empty, or

$$
\operatorname{dim} B N_{k}(r, a, \beta)=\mathrm{v} \cdot \operatorname{dim} B N_{k}(r, a, \beta) ;
$$

1. If $(k, r) \in D_{1}$, and at least one of the conditions $C_{1}, C_{2}$ and $C_{3}$ is satisfied, then $B N_{k}(r, a, \beta)$ is not empty, and

$$
\operatorname{dim} B N_{k}(r, a, \beta)=\mathrm{v} \cdot \operatorname{dim} B N_{k}(r, a, \beta) ;
$$

2. If $(k, r) \in D_{2}$ and $r \geq 2$, then $B N_{k}(r, a, \beta)$ is empty, even though v.dim $B N_{k}(r, a, \beta)>0$;

3. If $(k, r) \in D_{3}$, then $B N_{k}(r, a, \beta)$ is empty, as expected.

3.10 Conjecture: If Pic $S=\mathbb{Z} a$, then for any $(k, r) \in D_{0}$ the variety $B N_{k}(v)$ is not empty.

3.11 Remark. The second statement could not be made stronger: we give examples of situations in which $r=0$ or $r=1,(k, r) \in D_{2}$, and $B N_{k}(r, a, \beta)$ is not empty.

3.12 Remark. Let $k_{2}$ be the positive root of the equation

$$
2 g-k_{2}\left(k_{2}-\beta\right)=0
$$


The point $\left(k_{2}, 0\right)$ is on the boundary of $D_{2}$, as on the Figure 1 Note that $k_{1}$ satisfies the equality

$$
g-k_{1}\left(k_{1}-\beta\right)=0,
$$

i.e., for a genus $g$ curve $C$ and an integer $k=r+1$ one has v.dim $W_{d}^{r}(C)=$ $g-k(k-\beta)$, and

$$
k \leq k_{1} \text { iff v.dim } W_{d}^{r}(C) \geq 0
$$

Let $\pi: C \rightarrow T$ be a family of smooth genus $g$ curves, and let $\operatorname{Pic}_{C / T}^{d}$ be the relative Picard variety. One can define the relative Brill-Noether scheme $\left(W_{d}^{r}\right)_{C / T}$ over $T$ in such a way that the fiber of $\left(W_{d}^{r}\right)_{C / T}$ over a point $t \in T$ is isomorphic to the Brill-Noether subscheme $W_{d}^{r}\left(C_{t}\right)$ in the fiber $\left(\operatorname{Pic}_{C / T}^{d}\right)_{t} \simeq$ $\operatorname{Pic}^{d}\left(C_{t}\right)$. (Note that we do not expect $\left(W_{d}^{r}\right)_{C / T}$ to be flat over $T$.) It is natural to define

$$
\mathrm{v} \cdot \operatorname{dim}\left(W_{d}^{r}\right)_{C / T}=\operatorname{dim} T+\mathrm{v} \cdot \operatorname{dim} W_{d}^{r}\left(C_{t}\right)=\operatorname{dim} T+\rho(r, g, d)
$$

One would expect that if the family $C / T$ is generic enough, then

$$
\operatorname{dim}\left(W_{d}^{r}\right)_{C / T}=\mathrm{v} \cdot \operatorname{dim}\left(W_{d}^{r}\right)_{C / T}
$$

. In particular, if $C / T$ is generic enough and $\operatorname{dim} T=g$, as is the case when $T=|a|^{s}$, then one would expect

$$
\operatorname{dim}\left(W_{d}^{r}\right)_{C / T}=\mathrm{v} \cdot \operatorname{dim}\left(W_{d}^{r}\right)_{C / T}=g+(g-k(k-\beta))=2 g-k(k-\beta)
$$

It follows that $k_{2}$ can be defined by the property

$$
k=r+1 \leq k_{2} \text { iff } \operatorname{v} \cdot \operatorname{dim}\left(W_{d}^{r}\right)_{C /|a|^{s}} \geq 0,
$$

where $C /|a|^{s}$ is the universal curve over the linear system $|a|^{s}$. In particular, one would expect that for each $k$ such that $k_{1}<k=r+1<k_{2}$ the variety $W_{d}^{r}\left(C_{t}\right)$ is empty for a generic point $t \in T$ and that there is a codimension $|\rho(r, g, d)|$ subscheme in $T$ parametrizing points $t \in T$ such that $W_{d}^{r}\left(C_{t}\right)$ is not empty. In particular, one would expect that for $k_{1}<k=r+1<k_{2}$ the variety $\left(W_{d}^{r}\right)_{C /|a|^{s}}$ is not empty.

However, if $S$ is a K3 surface and $a \in \operatorname{Num} S$, one can not expect that the variety $\left(W_{d}^{r}\right)_{C /|a|^{s}}$ has the expected dimension, i.e., that the classifying map from $|a|^{s}$ to the moduli space of genus $g$ curves is of general position with respect to the stratification of $M_{g}$ by Brill-Noether special curves. (For example, we know that if one curve in the linear system $|C|$ is hyperelliptic, than all the curves in $|C|$ are hyperelliptic, and so on.) This discrepancy creates the region $D_{2}$ where the Brill-Noether loci are empty in the regular examples, even though their expected dimension is positive.

\section{The correspondence $\mathcal{A}_{r, r^{\prime}}$}

Let us fix $v \in K^{\prime}(S), v=\left(r, v_{1}, v_{2}\right)$, and an integer $r^{\prime}$ such that $0 \leq r^{\prime}<r$. Let $v^{\prime}=\left(r^{\prime}, v_{1}, v_{2}\right)$ and $l=r-r^{\prime}$. 


\subsection{Proposition.}

There is a coarse moduli space $A_{l}(v)$ classifying pairs $(E, V)$, where $E$ is a coherent sheaf on $S$ and $V \subset H^{0}(S, E)$ is a vector space of dimension $l$, which satisfy the following conditions:

1. $E$ is acceptable and $H$-stable,

2. $\operatorname{ch}(E)=v$,

3. the canonical "evaluation" map

$$
e_{E, V}: V \otimes \mathcal{O}_{S} \rightarrow E
$$

is monomorphic,

4. coker $e_{E, V}$ is acceptable and $H$-stable.

The proof of the existence of such a moduli space draws on the results of Le Potier and He Min (18, 19, 20, 21).

Given a scheme $X$ and morphisms $f: X \rightarrow Y$ and $g: X \rightarrow Z$, we say that we are given correspondence between $Y$ and $Z$, and denote this correspondence as $(X, f, g)$.

The definition of the moduli space $A_{l}(v)$ gives the correspondence $\mathcal{A}_{r, r^{\prime}}(v):=$ $\left(A_{l}(v), \pi_{1}, \pi_{2}\right)$ between $M(v)$ and $M\left(v^{\prime}\right)$,

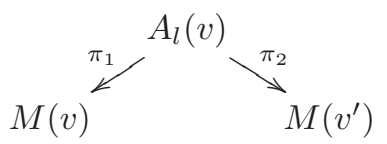

where $\pi_{1}([(E, V)])=[E]$ and $\pi_{2}([(E, V)])=$ coker $e_{E, V}$.

A point $[(E, V)] \in A_{l}(v)$ gives an exact sequence

$$
0 \rightarrow V \otimes \mathcal{O}_{S} \stackrel{e_{(E, V)}}{\rightarrow} E \rightarrow F \rightarrow 0
$$

Since $h^{1}\left(S, O_{S}\right)=0$, we have $h^{0}(S, F)=h^{0}(S, E)-l$.

Let $A_{l}^{k}(v)$ be the closed subscheme in $A_{l}(v)$ given by the condition $h^{0}(S, E) \geq$ $k$. Let $\left(A_{l}^{k}\right)^{o}(v)=A_{l}^{k}(v)-A_{l}^{k+1}(v)$, and let $B N_{k}^{o}(v):=B N_{k}(v)-B N_{k+1}(v)$. Restricting the correspondence $\mathcal{A}_{r, r^{\prime}}(v)$ to the locally closed subscheme $\left(A_{l}^{k}\right)^{o}(v)$ of $A_{l}(v)$, we get a correspondence $\mathcal{A}_{r, r^{\prime}}^{k, k^{\prime}}(v):=\left(\left(A_{l}^{k}\right)^{o}(v), \pi_{1}^{k}, \pi_{2}^{k}\right)$ :

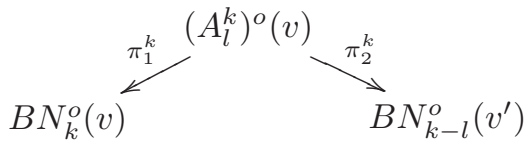

4.2 Lemma. For every $[E] \in B N_{k}(v)^{o}(v)$ the fiber $\left(\pi_{1}\right)^{-1}([E])$ is isomorphic to an open subscheme in the Grassmanian variety $\operatorname{Gr}\left(l, H^{0}(S, E)\right)$, and for every $[F] \in B N_{k-l}(v)^{o}(v)$ the fiber $\left(\pi_{2}\right)^{-1}([F])$ is isomorphic to an open subscheme in the Grassmanian variety $\operatorname{Gr}\left(l, \operatorname{Ext}_{S}^{1}\left(F, \mathcal{O}_{S}\right)\right)$. 
4.3 Remark. Let $C$ be a smooth algebraic curve, $\operatorname{Pic}^{d}(C)$ be the Picard variety of degree $d$ line bundles on $C$, and let $A_{1}(d)$ be the moduli space of pairs $(L, V)$, where $L \in \operatorname{Pic}^{d}(C)$ and $V$ is an one-dimensional vector subspace in $H^{0}(C, L)$. Existence of such a moduli space follows from the results of Le Potier, or it can be constructed directly. A point $(L, V) \in A_{1}(d)$ gives an exact sequence

$$
0 \rightarrow V \otimes \mathcal{O}_{C} \stackrel{e_{(L, V)}}{\rightarrow} L \rightarrow \operatorname{coker} e_{(L, V)} \rightarrow 0,
$$

which is isomorphic to the "adjunction" sequence

$$
0 \rightarrow \mathcal{O}_{C} \rightarrow \mathcal{O}_{C}(D) \rightarrow \mathcal{O}_{D}(D) \rightarrow 0
$$

where $D$ is the divisor of zeroes of a non-zero section $s \in V$. Since any effective divisor $D$ on $C$ can be written as a linear combination of points, $D=\sum n_{i}\left[p_{i}\right]$, the diagram

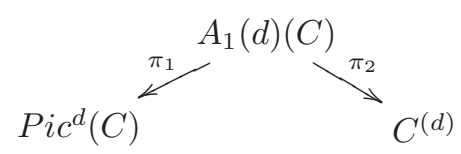

where $C^{(d)}$ is a symmetric power of the curve $C, \pi_{1}(L, V)=L$ and $\pi_{2}(L, V)=$ coker $e_{L, V}$, can be considered as an analogue of the correspondence 4.1] One can prove that $\pi_{2}$ is an isomorphism, and therefore the correspondence 4.2 "reduces" to the Abel-Jacobi morphism $\pi_{1} \pi_{2}^{-1}: C^{(d)} \rightarrow \operatorname{Pic}^{d}(C)$. This example lets us think about two particular correspondences, $\mathcal{A}_{r, 1}$ : $\operatorname{Hilb}^{d}(S) \longmapsto M(r, a, \beta)$ and $\mathcal{A}_{r, 0}: \quad \operatorname{Pic}^{2 g-2-d}(|a|) \longmapsto M(r, a, \beta)$, as two different analogs of the AbelJacobi correspondence in dimension two, corresponding to the fact that there are two types of subschemes on a surface: 0-cycles and curves.

\section{A few technical lemmas}

Our study of the correspondence $\mathcal{A}_{r, r^{\prime}}$ is based on the following six technical lemmas:

5.1 Lemma: Generic extensions are acceptable. Let $S$ be an algebraic surface, $F$ be an acceptable coherent sheaf on $S$, and let $W$ be a $k$-vector space. Let $\mathbb{A}$ be the affine scheme associated with the $k$-vector space $\operatorname{Ext}_{S}^{1}\left(F, W \otimes \mathcal{O}_{S}\right)$. The affine space $\mathbb{A}$ parametrizes extension classes of the form

$$
0 \rightarrow W \otimes \mathcal{O}_{S} \rightarrow E \rightarrow F \rightarrow 0
$$

We say that such an extension acceptable if $E$ is acceptable.

1. If $\operatorname{rk} F \geq 2$, i.e., $F$ is locally free, then all such extensions are acceptable;

2. if $\operatorname{rk} F=1$, i.e., $F=J_{\xi}(L)$, where $L$ is an ample invertible sheaf on $S$, $\xi$ is a simple effective 0 -subscheme on $S$, and the pair $\left(\xi, L+K_{S}\right)$ is CaleyBacharash, then there is a nonempty open subscheme $U \subset \mathbb{A}$ such that all the extensions parametrized by $U$ are acceptable; 
3. if $\operatorname{rk} F=0$, i.e., $F=\left(i_{C}\right)_{*}(B)$, then

(a) If $\operatorname{dim} W=1$, then all the extensions parametrized by $\mathbb{A}-\{0\}$ are acceptable;

(b) If $\operatorname{dim} W \geq 2$, and $A:=N_{C / X} B^{-1}$ is a globally generated line bundle on $C$, then there is a nonempty open subscheme $U \subset \mathbb{A}$ such that every extension class $e \in U$ is acceptable;

(c) In particular, let $\operatorname{dim} W \geq 2$, and assume that $A=N_{C / X} B^{-1}$ is globally generated and that $\operatorname{dim} W=H^{0}(C, A)$. Let $\alpha_{e}: W^{\vee} \rightarrow$ $H^{0}(C, A)$ be the image of $e$ under the isomorphism

$$
\operatorname{Ext}_{S}^{1}\left(i_{*} B, W \otimes \mathcal{O}_{S}\right) \simeq H^{1}(C, W \otimes A) \simeq \operatorname{Hom}\left(W^{\vee}, H^{0}(C, A)\right)
$$

If $\alpha_{e}$ is an isomorphism, then $e$ is acceptable.

5.2 Lemma : Generic evaluation maps in the globally generated case are monomorphic. Let $X$ be a reduced irreducible scheme, $E$ be a globally generated locally free sheaf on $X$, and $l \leq \mathrm{rk} E$. Then there is a nonempty open subset in the Grassmanian variety $G r\left(l, H^{0}(E)\right)$ parametrizing vector subspaces $V \subset H^{0}(X, E)$ for which the evaluation map $e_{(E, V)}: V \otimes \mathcal{O}_{X} \rightarrow E$ is monomorphic.

5.3 Lemma: Generic factors in the globally generated case are acceptable. Let $S$ be a smooth algebraic surface, chark $=0$, let $E$ be a globally generated locally free sheaf on $S$, and let $1 \leq l \leq \operatorname{rk} E$. Then there is a nonempty open subset in the Grassmanian variety $\operatorname{Gr}\left(l, \bar{H}^{0}(E)\right)$ parametrizing vector subspaces $V \subset H^{0}(S, E)$ for which the cokernel of the evaluation map

$$
e_{V}: H^{0}(S, E) \underset{k}{\otimes} \mathcal{O}_{S} \rightarrow E
$$

is acceptable.

5.4 Lemma: Stability of extensions. Let $(S, H)$ be a polarized algebraic surface, and let $F$ be an $H$-stable acceptable sheaf on $S$. Assume that we are given an extension

$$
0 \rightarrow W \otimes \mathcal{O}_{X} \rightarrow E \rightarrow F \rightarrow 0
$$

Let $e$ be the class of this extension in $\operatorname{Ext}^{1}\left(F, W \otimes \mathcal{O}_{X}\right)$, and let $\alpha_{e}$ be the image of $e$ under the isomorphism $\operatorname{Ext}^{1}\left(F, W \otimes \mathcal{O}_{X}\right) \stackrel{\sim}{\rightarrow} \operatorname{Hom}\left(W^{\vee}, \operatorname{Ext}^{1}(F, \mathcal{O})\right)$.

1. If $\alpha_{e}$ is not injective, then $E$ is not $H$-stable;

2. If $\alpha_{e}$ is injective, $F$ is acceptable, and either

(a) $\operatorname{Pic} S \simeq \mathbb{Z} \cdot c_{1}(E)$, or

(b) $\left(c_{1}(F), H\right)=1$,

then $E$ is $H$ - stable. 
5.5 Lemma: Stability of factors. Let $(S, H)$ be a polarized algebraic surface, let $E$ be an $H$-stable coherent sheaf on $S$, and assume that we are given an exact sequence of the form

$$
0 \rightarrow W \otimes \mathcal{O}_{S} \rightarrow E \rightarrow F \rightarrow 0
$$

where $F$ is a torsion-free sheaf. Assume further that either Pic $S \simeq \mathbb{Z} \cdot H$ and $c_{1}(E)=H$, or that $\left(c_{1}(E), H\right)=1$. Then $F$ is $H$-stable.

5.6 Lemma: The correspondence preserves the property of being globally generated. Let $X$ be a scheme satisfying $h^{0}\left(X, \mathcal{O}_{X}\right)=1$, and assume that we are given an extension of coherent sheaves

$$
0 \rightarrow \mathcal{O}_{X} \rightarrow E \rightarrow F \rightarrow 0
$$

If $E$ is globally generated, then $F$ is globally generated. If $F$ is globally generated and $H^{1}\left(X, \mathcal{O}_{X}\right)=0$, then $E$ is globally generated.

The proof of lemmas 5.1- 5.6 will be given in 22.

\section{Numerical structure of the correspondence $\mathcal{A}$ on a K3 surface}

Let $S$ be a K3-surface. Consider the map $p$ from the set of the isomorphism classes of acceptable coherent sheaves on $S$ to the free Abelian group $P=\mathbb{Z}^{2}$, $p(E)=\left(h^{0}(S, E), \operatorname{rk}(E)\right)$. We denote the coordinates on $P$ as $(K, R)$.

If $F$ is an acceptable sheaf on $S, v=\operatorname{ch}(F)=(r, a, \beta)$ and $\epsilon(v)=(r, g, d)$, we have

$$
\beta=g-1-d
$$

and define

$$
\alpha=-\beta=d+1-g
$$

By the Riemann-Roch formula, we have $\chi(S, F)=2 r+\beta$.

If $F$ is an acceptable sheaf on $S$ satisfying $h^{1}(S, F)=0$ and $h^{2}(S, F)=0$, then $h^{0}(S, F)=\chi(S, F)=2 r+\beta$, and $p(E)$ is a point on the line $l(\beta)$ : $(K=2 R+\beta)$ in $P_{\mathbb{R}}=P \otimes \mathbb{R}$. I.e., $p$ maps the maximal Brill-Noether strata $B N_{\chi(v)}(v)^{o}$ to the line $l=l(\beta)$, as on the Figure 2

Note that the correspondences $\mathcal{A}_{r, r^{\prime}}^{k, k^{\prime}}$ acts along the lines $l^{\prime}(c):(K-R=c)$ in $P_{\mathbb{R}}$. Note also that for every $\left(k^{\prime}, r^{\prime}\right) \in P$ the line $l^{\prime}\left(k^{\prime}-r^{\prime}\right)$ contains $\left(k^{\prime}, r^{\prime}\right)$ and intersects the line $l(\beta)$ at some point $(k, r)$, i.e., for every $(k, r) \in P$ there is a $\left(k^{\prime}, r^{\prime}\right) \in l(\beta)$ and a correspondence $\mathcal{A}_{r, r^{\prime}}^{k, k^{\prime}}$ between $B N_{k^{\prime}}\left(r^{\prime}, a, \beta\right)$ and the maximal Brill-Noether loci $B N_{k}(r, a, \beta)$, as on the Figure 3

Thus the correspondence $\mathcal{A}$ relates the geometry of Brill-Noether loci on the lines $l^{\prime}$. We use this correspondence twice, first time to derive certain properties of the maximal Brill-Noether loci $B N_{2 r+\beta}(r, a, \beta)$ and second time to relate $B N_{2 r+\beta}(r, a, \beta)$ and a given Brill-Noether variety $B N_{k^{\prime}}\left(r^{\prime}, a, \beta\right)$ in order to establish that the latter behave in the way predicted by the formula 3.2 in a certain domain. 

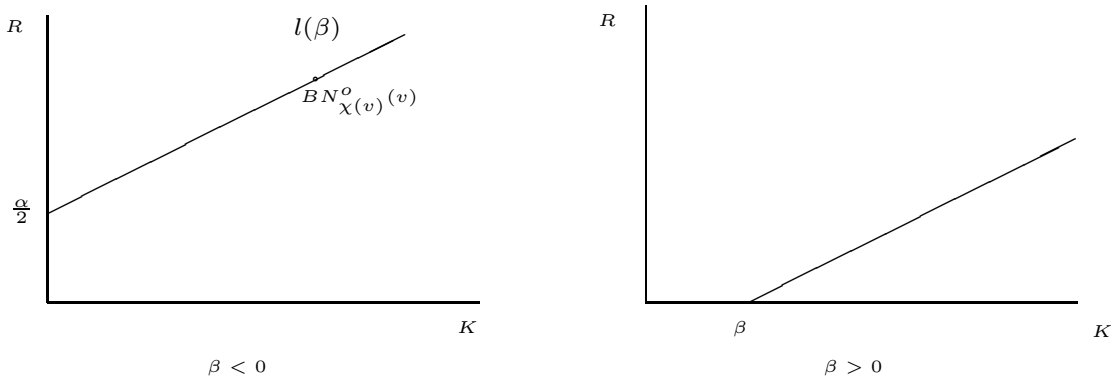

Figure 2: Geography of the maximal Brill-Noether strata
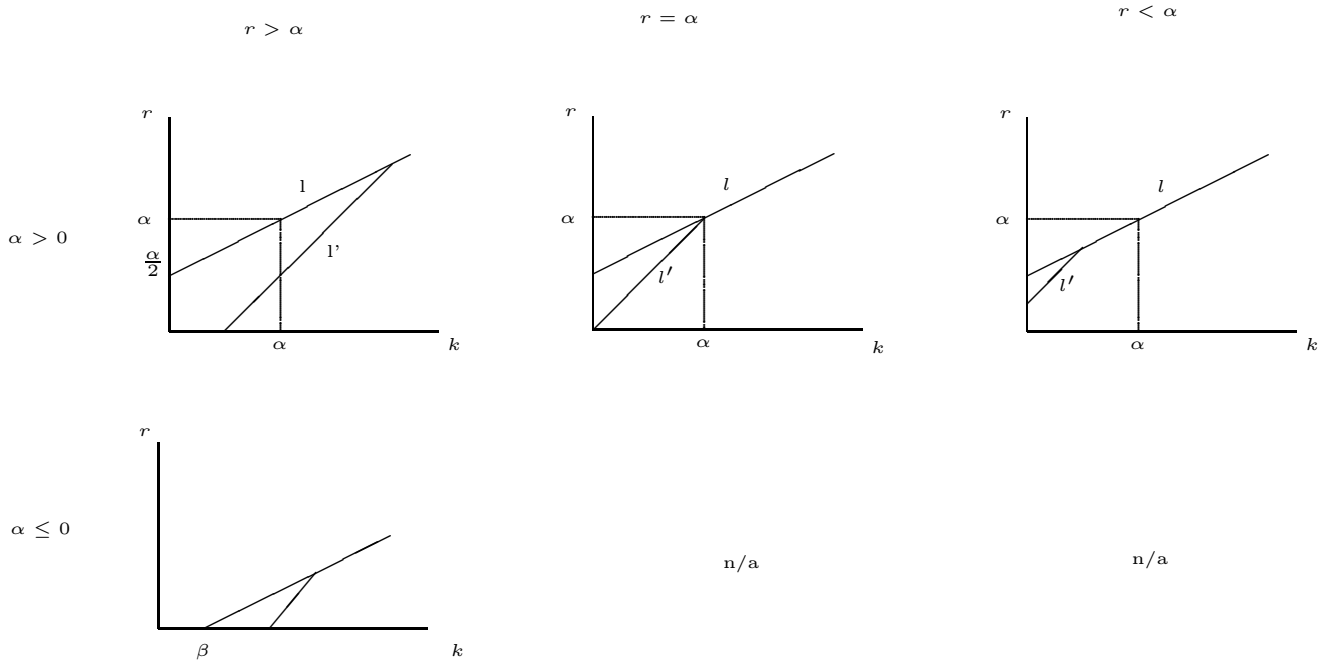

$\mathrm{n} / \mathrm{a}$

$\mathrm{n} / \mathrm{a}$

Figure 3: Relative position of the lines $l$ and $l^{\prime}$

\section{Four special cases.}

Let $v=(r, a, \beta)$ and $v^{\prime}=\left(r^{\prime}, a, \beta\right), v, v^{\prime} \in K^{\prime}(S)$. We will be studying the correspondence $\mathcal{A}_{r, r^{\prime}}^{k, k^{\prime}}$. The following four cases are particularly interesting:
(a) $p(v) \in l, r^{\prime}=0$;
(b) $p(v) \in l, r^{\prime}$ is any integer such that $0 \leq r^{\prime}<r$;
(c) $r^{\prime}=0$;
(d) $r^{\prime}=1$.

(Note that (a) is a special case of (b) and (c).) 


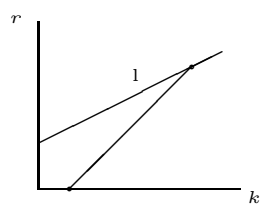

b

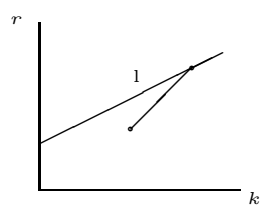

C

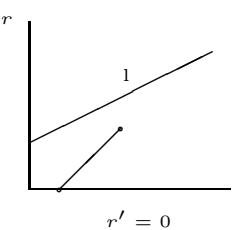

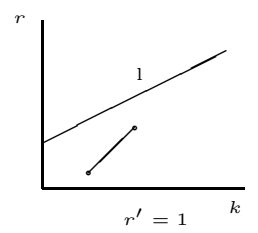

Figure 4: Four special cases

These cases are illustrated by the Figure 4

We use cases (a) and (b) to prove our main theorem, and cases (c) and (d) to establish certain birational properties of the birational geometry of the moduli spaces.

There is a fifth interesting case, one with $r=1$ and $r^{\prime}=0$, which relates the geometry of the Hilbert scheme of points on $S$ and the relative Picard variety for curves in the linear system $|a|$. We do not consider it in this text (see 22] for more details).

We will analyze these four situations case-by case.

\subsection{Case (a)}

Let us fix $v=(r, a, \beta), r \geq 2$, and let $v^{\prime}=(0, a, \beta)$. Let $k=\chi(v)=2 r+\beta$ and $k^{\prime}=r+\beta$. Assume that $k \geq r$, i.e., $(k, r) \notin D_{0}$. It follows that $k^{\prime} \geq 0$ and $r \geq \alpha$.

Consider the correspondence $\mathcal{A}_{r, 0}^{2 r+\beta, r+\beta}$,

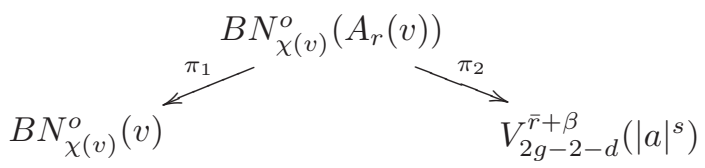

as illustrated on the Figure $[$ in the case $\beta<0$.

Note that for a curve $C \in|a|^{s}$ there is a Serre duality isomorphism $V_{2 g-2-d}^{\bar{r}+\beta}(C) \stackrel{\sim}{\rightarrow}$ $V_{d}^{\bar{r}}(C)$.

If $B N_{\chi(v)}^{0}(v)$ is not empty, then the fiber $\pi_{1}^{-1}([E])$ over $[E] \in B N_{\chi(v)}^{0}(v)$ is isomorphic to an open subscheme in the Grassmanian variety $\operatorname{Gr}\left(r, H^{0}(S, E)\right) \simeq$ $\operatorname{Gr}(r, 2 r+\beta)$ of dimension $r(r+\beta)$. (Note that $r+\beta \geq 0$ ). If $V_{2 g-2-d}^{\bar{r}+\beta}\left(|a|^{s}\right)$ is not empty, then the fiber $\pi_{2}^{-1}\left(\left(i_{C}\right)_{*}(B)\right)$ over a point $(C, B) \in V_{2 g-2-d}^{\bar{r}+\beta}\left(|a|^{s}\right)$ is isomorphic to an open subscheme in the Grassmanian variety $\operatorname{Gr}\left(r, \operatorname{Ext}_{S}^{1}\left(i_{*} B, \mathcal{O}_{S}\right)\right) \simeq$ $\operatorname{Gr}\left(r, H^{0}(C, A)\right) \simeq \operatorname{Gr}(r, r) \simeq \operatorname{Spec} k$, where $A=K_{C} B^{-1}$. This proves that $\pi_{2}$ is an embedding. Note that $h^{0}(C, A)=h^{1}(C, B)=h^{0}(C, B)-\chi(C, B)=$ $r+\beta-\beta=r$.

7.1 Lemma.Assume that the following conditions are satisfied: 


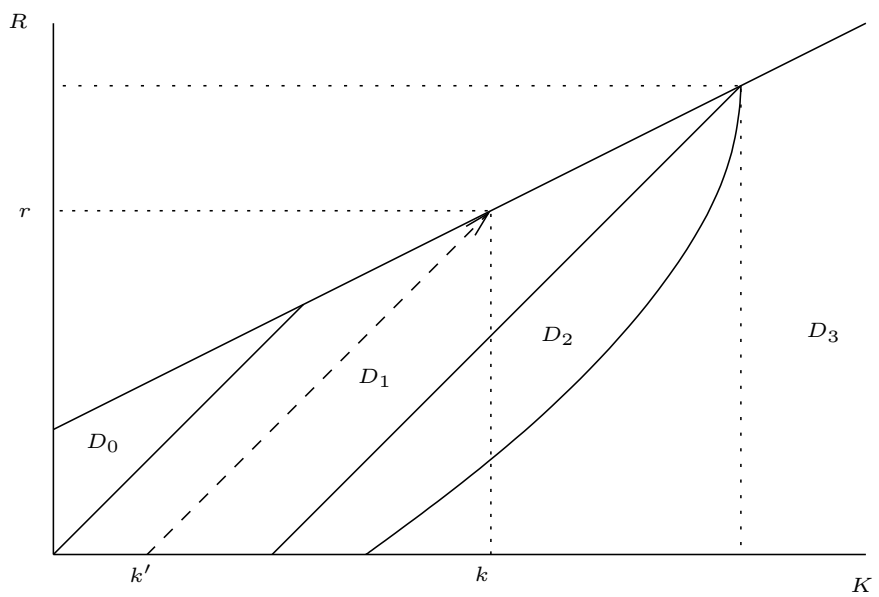

Figure 5: Geography in the case (a).

1. $\rho(\bar{r}, g, d) \geq 0$, i.e., $(k, r) \in D_{1}$,

2. There is a globally generated line bundle $A \in V_{d}^{\bar{r}}\left(|a|^{s}\right)$, and

3. Either Pic $S=\mathbb{Z} a$, or $(a, H)=1$.

Then $B N_{2 r+\beta}^{o}(r, a, \beta)$ is not empty. Moreover, if $\left(V_{d}^{\bar{r}}\right)^{\prime \prime}\left(|a|^{s}\right)$ is not empty, then $B N_{2 r+\beta}^{o}(r, a, \beta)$ contains a globally generated vector bundle.

Proof: By the assumption, there is a $C \in|a|^{s}$ and a globally generated line bundle $A \in V_{d}^{\bar{r}}(C)$. Let $B=K_{C} A^{-1}$, and let $W$ be a vector space of dimension $r$. Since

$$
\operatorname{dim}_{k} \operatorname{Ext}_{S}^{1}\left(\left(i_{C}\right)_{*} B, \mathcal{O}_{S}\right)=\operatorname{dim}_{k} H^{0}(C, A)=r,
$$

there is a unique (up to an automorphism of $W$ ) extension class $e$ of the form

$$
0 \rightarrow W \otimes \mathcal{O}_{S} \rightarrow E \rightarrow i_{*} B \rightarrow 0
$$

such that the induced map $\alpha_{e}: W^{\vee} \rightarrow \operatorname{Ext}_{S}^{1}\left(i_{*} B, \mathcal{O}_{S}\right)$ is an isomorphism. By Lemma 5.1] $E$ is locally free. Since either Pic $S=\mathbb{Z} a$ or $(a, H)=1$, Lemma 5.4 implies that $E$ is $H$-stable. Thus $E \in B N_{\chi(v)}^{o}(v)$. Moreover, if $B$ is globally generated, then, by Lemma [5.6 $E$ is globally generated, which proves the lemma.

Our previous considerations imply the following

7.2 Lemma: If Pic $S=\mathbb{Z} a$ or $(a, H)=1$, then $\pi_{2}$ is an isomorphism of $B N_{\chi(v)}^{o}\left(A_{r}(v)\right)$ unto $\left(V_{2 g-2-d}^{\bar{r}+\beta}\right)^{\prime}\left(|a|^{s}\right)$. 
In particular, the correspondence $\mathcal{A}_{r, 0}^{2 r+\beta, r+\beta}$ gives a morphism

$$
L:\left(V_{2 g-2-d}^{\bar{r}+\beta}\right)^{\prime}\left(|a|^{s}\right) \rightarrow B N_{\chi(v)}^{o}(v)
$$

7.3 The construction above is equivalent to the construction given by Lazarsfeld in 10, as we will now explain.

Let $(C, A) \in V_{d}^{\bar{r}}\left(|a|^{s}\right)$, and assume that $A$ is globally generated. The canonical evaluation map $H^{0}(C, A) \otimes_{k} \mathcal{O}_{C} \rightarrow A$ induces an epimorphic map $H^{0}(C, A) \otimes_{k} \mathcal{O}_{S} \rightarrow\left(i_{C}\right)_{*} A$; denoting its kernel as $F$, we get an exact sequence of $\mathcal{O}_{S}$-modules

$$
0 \rightarrow F \rightarrow H^{0}(C, A) \underset{k}{\otimes} \mathcal{O}_{S} \rightarrow i_{*} A \rightarrow 0
$$

Note that $F$ is locally free since locally it is a kernel of an epimorphism $R^{n} \rightarrow$ $R / f \rightarrow 0$. By assumptions on $A$ we have $\operatorname{dim}_{k} H^{0}(C, A)=r$. Applying the functor $\mathcal{H o m}_{S}\left(\cdot, \mathcal{O}_{S}\right)$ to the exact sequence above, we get an extension

$$
0 \rightarrow H^{0}(C, A)^{\vee} \underset{k}{\otimes} \mathcal{O}_{S} \rightarrow F^{\vee} \rightarrow i_{*} B \rightarrow 0
$$

The long corresponding long exact sequence

$$
\begin{gathered}
0 \rightarrow H^{0}(C, A)^{\vee} \rightarrow H^{0}\left(S, F^{\vee}\right) \rightarrow H^{0}(C, B) \rightarrow 0, \\
0 \rightarrow H^{1}\left(S, F^{\vee}\right) \rightarrow H^{1}(C, B) \stackrel{\delta}{\rightarrow} H^{0}(C, A)^{\vee} \rightarrow H^{2}\left(S, F^{\vee}\right) \rightarrow 0
\end{gathered}
$$

gives $h^{0}\left(S, F^{\vee}\right)=h^{0}(C, A)+h^{0}(C, B)=r+(r+\beta)=\chi(v)$. One can check that $\delta$ is the Serre duality isomorphism, and therefore $h^{1}\left(S, F^{\vee}\right)=h^{2}\left(S, F^{\vee}\right)=0$.

If Pic $S=\mathbb{Z} a$ or $(C, H)=1$, then $F^{\vee}$ is stable by lemma 5.4

It follows that $F^{\vee} \in B N_{\chi(v)}^{o}(r, a, \beta)$. Thus we have a morphism

$$
\begin{aligned}
L:\left(V_{2 g-2-d}^{\bar{r}+\beta}\right)^{\prime}\left(|a|^{s}\right) & \rightarrow B N_{\chi(v)}^{o}(r, a, \beta), \\
(C, B) & \mapsto F^{\vee}
\end{aligned}
$$

It follows that the case (a) of the correspondence $\mathcal{A}$ restores the construction of Lazarsfeld (10]).

\subsection{Case (b) and proof of the main theorem}

Let us fix $v=(r, a, \beta), v^{\prime}=\left(r^{\prime}, a, \beta\right), r \geq 2,0 \leq r^{\prime}<r$, and $k^{\prime} \geq \chi\left(v^{\prime}\right)$. Let $k=\chi(v)=2 r+\beta$. Assume that $k \geq r$, i.e., $(k, r) \notin D_{0}$.

Consider the correspondence $\mathcal{A}_{r, r^{\prime}}^{\chi(v), k^{\prime}}$,

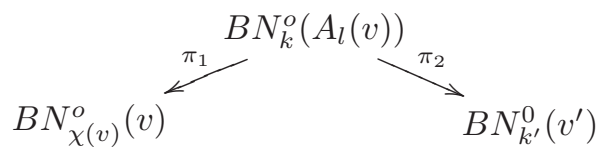

as illustrated by the Figure [6 We can assume 


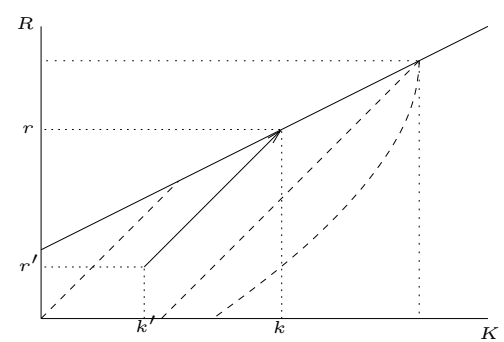

$\beta<0$

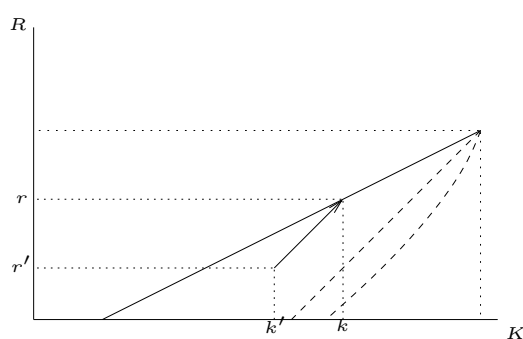

$\beta>0$

Figure 6: Geography in the case (b).

$$
\rho(\bar{r}, g, d) \geq 0
$$

i.e., $(k, r)$ and $\left(k^{\prime}, r^{\prime}\right) \in D_{1}$, since otherwise otherwise $M(r, a, \beta)$ and all the varieties in the correspondence 7.1 are empty.

Since $r-r^{\prime}=k-k^{\prime}$ and $k=2 r+\beta$, we have $r=k^{\prime}-r^{\prime}-\beta$ and $l=r-r^{\prime}=$ $k^{\prime}-2 r^{\prime}-\beta$.

Now, if we are given some $v^{\prime}=\left(r^{\prime}, a, \beta\right)$ and $k^{\prime} \geq \chi\left(v^{\prime}\right)=2 r^{\prime}+\beta$, we define $r$ by the formula given above, we can include $B N_{k^{\prime}}\left(v^{\prime}\right)$ in the correspondence of the form 7.1

\subsection{Proof of the main theorem:}

We start by proving part 1 . Let $(k, r) \in D_{1}$, and assume that $\left(V_{d}^{\bar{r}}\right)^{\prime \prime}\left(|a|^{s}\right)$ is not empty. Let $(C, B) \in\left(V_{d}^{\bar{r}}\right)^{\prime \prime}\left(|a|^{s}\right)$. By Lemma 7.1 the scheme $B N_{\chi(v)}^{0}(M(v))$ is not empty and contains a globally generated vector bundle. Now, by Lemmas 5.2 5.3 and 5.5 the variety $B N_{k}^{o}\left(A_{l}(v)\right)$ is not empty, and thus $B N_{k^{\prime}}\left(v^{\prime}\right)$ is not empty.

Now the variety $B N_{k}^{o}(v)$ is a nonempty open subscheme in $M(v)$, and thus

$$
\operatorname{dim} B N_{\chi(v)}^{0}(v)=\operatorname{dim} M(v)=2 \rho(\bar{r}, g, d)
$$

Let $B N_{\chi(v)}^{o, g g}(v)$ be an open subscheme in $B N_{\chi(v)}^{o, g g}(v)$ parametrizing globally generated sheaves; we have just proved that it is not empty. The lemmas 5.2 5.3 and 5.5 imply that the fiber of $\pi_{1}$ over a point of $B N_{\chi(v)}^{o, g g}(v)$ is isomorphic to a nonempty open subscheme in the Grassmanian variety $\operatorname{Gr}(l, 2 r+\beta)$ of dimension $l(2 r+\beta-l)$. (Note that since $l \leq 2 r+\beta$, this number is not negative). In particular, $\pi_{1}$ is epimorphic over $B N_{\chi(v)}^{o, g g}(v)$.

Let us study the fibers of $\pi_{2}$. The lemmas 5.1 and 5.4 imply that

1. If $r^{\prime} \geq 2$, then $\pi_{2}$ is epimorphic;

2. If $r^{\prime}=1$, then $\pi_{2}$ is epimorphic over a subscheme of simple CaleyBacharash 0-cycles; 
3. If $r^{\prime}=0$ and $l=1$, then $\pi_{2}$ is epimorphic, and

4. If $r^{\prime}=0$ and $l \geq 2$, then $\pi_{2}$ is epimorphic over $\left(V_{2 g-2-d}^{\bar{r}+\beta}\right)^{\prime}\left(|a|^{s}\right)$.

For the proof of part one of the main theorem, we need to consider only the case $r^{\prime} \geq 2$, when $\pi_{2}$ is epimorphic.

The fiber of $\pi_{2}$ over a point $E^{\prime} \in B N_{k^{\prime}}\left(M\left(v^{\prime}\right)\right)$ is isomorphic to an open subscheme in the Grassmanian variety $\operatorname{Gr}\left(l, \operatorname{Ext}_{S}^{1}\left(E^{\prime}, \mathcal{O}_{S}\right)\right) \simeq \operatorname{Gr}\left(l, H^{1}\left(S, E^{\prime}\right)^{\vee}\right) \simeq$ $\operatorname{Gr}\left(l, h^{1}\left(S, E^{\prime}\right)\right)$. It is easy to see geometrically that $h^{1}\left(S, E^{\prime}\right)=l$, and it follows that $\pi_{2}$ is an isomorphism. It follows that the correspondence $\mathcal{A}$ in the case (b) gives a morphism

$$
\pi_{2}^{-1} \pi_{1}: \quad B N_{k}^{o}\left(r^{\prime}, a, \beta\right) \rightarrow B N_{2 r+\beta}^{0}(r, a, \beta),
$$

where $r=k^{\prime}-r^{\prime}-\beta$, as denoted with an arrow on the Figure 6

Now counting of dimensions gives

$$
\begin{aligned}
& \operatorname{dim} B N_{k^{\prime}}\left(M\left(v^{\prime}\right)\right)=\operatorname{dim} M(v)+\operatorname{dim}\left(\text { fiber of } \pi_{1}\right)= \\
& =2(g-r(r-\alpha))+l(2 r+\beta-l)= \\
& =2\left(g-r^{\prime}\left(r^{\prime}-\alpha\right)\right)-k^{\prime}\left(k^{\prime}-\left(2 r^{\prime}+\beta\right)\right)=\operatorname{dim} M\left(v^{\prime}\right)-k^{\prime}\left(k^{\prime}-\chi\left(v^{\prime}\right)\right)= \\
& =\mathrm{v} \cdot \operatorname{dim} B N_{k^{\prime}}\left(v^{\prime}\right) \text {. }
\end{aligned}
$$

The proof of the part 0 of the main theorem is parallel, except that our study in the case (a) in this case does not guaranty that $B N_{k}\left(A_{l}^{o}(v)\right)$ is not empty.

To prove parts 2 and 3 of the main theorem, assume that $\left(k^{\prime}, r^{\prime}\right) \in D_{2} \cup D_{3}$. Assume that $B N_{k}^{\prime}\left(v^{\prime}\right)$ is not empty. Then, by Lemmas [5.1] and [5.4 the varieties $B N_{k}^{0}\left(A_{l}(v)\right)$ and thus $B N_{k}^{o}(v)$ are not empty, which contradicts to the Mukai theorem, since whenever $M(r, a, \beta)$ is not empty, the dimension $\operatorname{dim} M(r, a, \beta)=$ $2 \rho(\bar{r}, g, d)$ is not negative.

\subsection{Case (c)}

Let us fix $v=(r, a, \beta) \in K^{\prime}(S)$ and $k \in \mathbb{Z}$ such that $r \geq 2$ and $k \geq \max (\chi(v), r)=$ $\max (2 r-\beta, r)$. Consider the correspondence $\mathcal{A}_{r, 0}^{k, k-r}$,

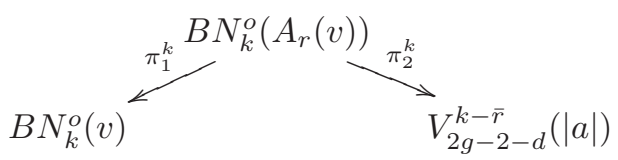

Let $\left(k_{0}, r_{0}\right)$ be the intersection point of the lines $l(\beta)$ and $l^{\prime}(k, r)$, as on the Figure [7. We have $r_{0}=k-r-\beta$. The results of [7.1] and 7.2 ensure that if

1. $(k, r) \in D_{1}$

2. The variety $\left(V_{d}^{\bar{r}_{0}}\left(|a|^{s}\right)\right)^{\prime \prime}$ is not empty, and

3. Pic $S=\mathbb{Z} a$ or $(a, H)=1$, 


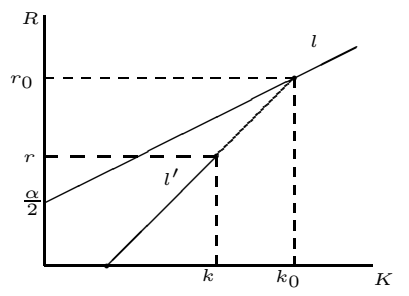

Figure 7: Geography in the case (c).

then all the terms in the correspondence $\mathcal{A}_{r, 0}^{k, k-r}$ are not empty and the morphisms $\pi_{1}^{k}$ and $\pi_{2}^{k}$ are dominant.

Let us fix $v$ and vary $k \in \mathbb{Z}, k \geq \chi(v)$. As one can see from the Figure 8 there are three combinatorial variants, when $r<\alpha, r=\alpha$ and $r>\alpha$.

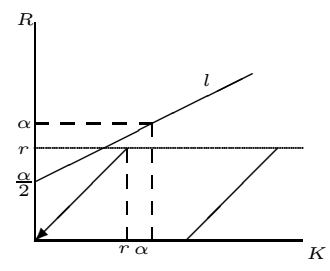

$r<\alpha$

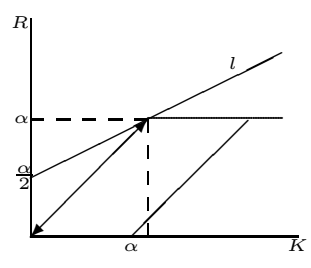

$r=\alpha$

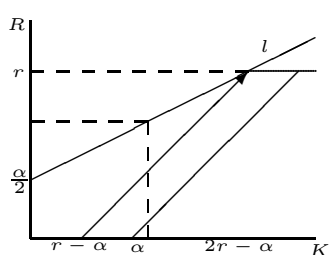

$r>\alpha$

Figure 8: Geography in the case (c), three variants

If $r<\alpha$, then the tom term in the correspondence $\mathcal{A}_{r, 0}^{k, k-r}$ is empty for $k<r$, and all the terms are nonempty if $k \geq r$. Additionally, if $k=r$, then $\pi_{1}^{r}$ is an isomorphism over a subscheme of globally generated vector bundles in $B N_{r}(v)$, and thus $\pi_{1}^{r}$ is a birational isomorphism and the correspondence $\mathcal{A}_{r, 0}^{r, 0}$ gives a rational map $B N_{r}(r, a, \beta) \rightarrow \operatorname{Pic}^{d}\left(|a|^{s}\right)$, marked with an arrow on the Figure 8 If $r=\alpha$, the correspondence $\mathcal{A}_{r, 0}^{r, 0}$ gives a birational isomorphism

$$
M(r, a,-r) \stackrel{\text { bir }}{\simeq} \operatorname{Pic}^{2 g-2-d}(|a|)
$$

where $d=g+r=\frac{a^{2}+2}{2}+r$.

If $r>\alpha$, the map $\pi_{2}^{k}$ is a birational isomorphism for $k=\chi(v)$, and the correspondence $\mathcal{A}_{r, 0}^{2 r-\alpha, r-\alpha}$ gives a rational morphism $V_{2 g-2-d}^{\bar{r}-\alpha}(|a|) \rightarrow M(v)$, which is denoted by an arrow on the Figure 8

It follows that

1. If $r<\alpha$ then the moduli space $M(v)$ contains a Brill-Noether subscheme $B N_{r}(v)$ which is birationally isomorphic to a Grassmanian fibration over the relative Picard scheme $\operatorname{Pic}^{2 g-2-d}(|a|)$; 
2. If $r=\alpha$ then the moduli space $M(v)$ is birationally isomorphic to the relative Picard variety $\mathrm{Pic}^{2 g-2-d}(|a|)$, and

3. If $r>\alpha$, then the relative Picard variety $\operatorname{Pic}^{2 g-2-d}(|a|)$ contains a relative Brill-Noether subscheme $W_{2 g-2-d}^{\bar{r}-\alpha}(|a|)$ which is birationally isomorphic to a Grassmanian fibration over the moduli space $M(v)$.

\subsection{Case (d)}

Let us fix $v=(r, a, \beta)$ such that $r \geq 2$ and $k \geq \max (\chi(v), r-1)$. The fibers of the map $\pi_{1}^{k}$ in the correspondence $\mathcal{A}_{r, 1}^{k, k-(r-1)}$

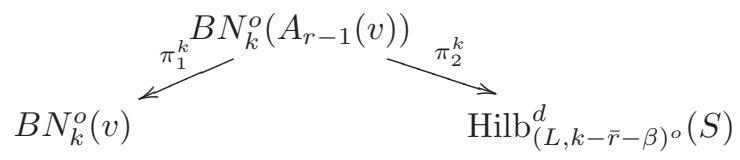

are isomorphic to open subschemes in the Grassmanian $\operatorname{Gr}(r-1, k)$, and the fibers of $\pi_{2}^{k}$ are isomorphic to open subschemes in the Grassmanian $\operatorname{Gr}(r-1, k-$ $r+\alpha-1$ ). (Note that $r-1 \leq k-r+\alpha-1$, and $r-1=k-r+\alpha-1$ only if $k=\chi(v))$.

Let $\left(k_{0}, r_{0}\right)$ be the intersection point of $l(\beta)$ and $l^{\prime}(k, r)$. We have $r_{0}=$ $k-r-\beta$. Lemma [7.] implies that if

1. $(k, r) \in D_{1}$,

2. The variety $\left(V_{d}^{\bar{r}_{0}}\left(|a|^{s}\right)\right)^{\prime \prime}$ is not empty, and

3. Pic $S=\mathbb{Z} a$ or $(a, H)=1$,

then $B N_{\chi_{0}}\left(v_{0}\right)$ is not empty and contains a globally generated vector bundle, and Lemmas 5.2 and $\left[5.3\right.$ imply that $\operatorname{Hilb}_{(L, k-\bar{r}-\beta)^{o}}^{d}(S)$ is not empty. One can see that the morphisms $\pi_{1}^{k}$ and $\pi_{2}^{k}$ are dominant.

There are three combinatorial possibilities, depending on whether $r<\alpha-1$, $r=\alpha-1(d=r+g)$ or $r>\alpha-1$, as one can see on the Figure 9

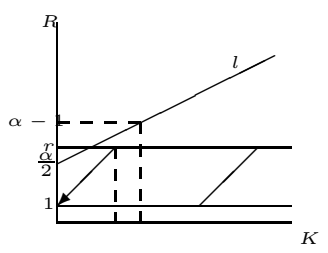

$r<\alpha-1$

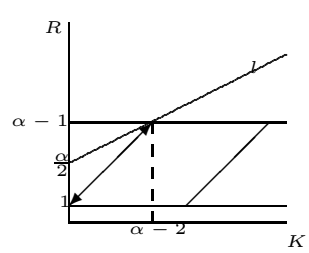

$r=\alpha-1$

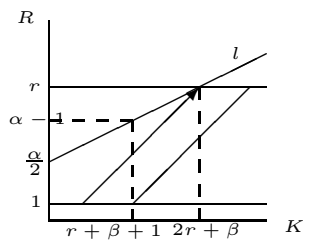

$r>\alpha-1$

Figure 9: Geography in the case (d), three variants 
In the first case $k \geq r-1$, the morphism $\pi_{2}^{k}$ has a positive relative dimension and the morphism $\pi_{1}^{k}$ is a birational isomorphism for $k=r-1$ and has a positive relative dimension for $k>r-1$. It follows that for $k=r-1$ the correspondence $\mathcal{A}_{r, 1}^{k}$ gives a rational map

$$
B N_{r-1}(r, a, \beta) \rightarrow \operatorname{Hilb}^{d}(S),
$$

denoted with an arrow on the picture above.

If $r=\alpha-1$, then $k \geq r-1$ and for $k=r-1$ both $\pi_{1}^{k}$ and $\pi_{2}^{k}$ are birational isomorphisms, and thus we get the birational isomorphism

$$
M(r, a,-r-1) \stackrel{\text { bir }}{\simeq} \operatorname{Hilb}^{d}(S)
$$

where $d=g+r=\frac{a^{2}+2}{2}+r$.

[In the case $r=2$ this statement was proved by Lothar Goettsche.]

If $r>\alpha-1$, then $k \geq \chi(v)$, the map $\pi_{1}^{k}$ has a positive relative dimension, and $\pi_{2}^{k}$ is a birational isomorphism for $k=2 r+\beta$ and has a positive relative dimension for $k>2 r+\beta$. It follows that for $k=2 r+\beta(\delta(\xi, L)=r-1)$ the correspondence gives a rational morphism $\operatorname{Hilb}_{L, r-1}^{d}(S) \rightarrow M(v)$, which is denoted by an arrow on the picture above.

Summarizing the results, we can see that for $r<\alpha-1$ the moduli space $M(v)$ contains a Brill-Noether subscheme which is birational to a Grassmanian fibration over the Hilbert scheme $\operatorname{Hilb}^{d}(S)$, for $r=\alpha-1$ the moduli space $M(v)$ is birationally isomorphic to the Hilbert scheme $\operatorname{Hilb}^{d}(S)$, and for $r>\alpha-1$ the Hilbert scheme $\operatorname{Hilb}^{d}(S)$ contains a subscheme of special 0-cycles which is birational to a Grassmanian fibration over the moduli space $M(v)$.

\section{Examples.}

\subsection{Example: $g=3, d=3$}

Let $(S, a)$ be a polarized K3 surface of genus $g=3$, and let $d=3$. Note that $M(0, a, \beta)=M(0, a,-1) \subset \operatorname{Pic}^{1}(|a|)$, since that for $(C, B) \in M(0, a, \beta)$ we have $\operatorname{deg} B=2 g-2-d=1$.

The formula 3.2 gives the following expected dimensions of the Brill-Noether loci in various $M(r, a,-1)$ :

\begin{tabular}{l|lllll}
$\mathrm{r}$ & & & & & \\
2 & & & & 2 & \\
1 & & 6 & 4 & 0 & \\
0 & 6 & 4 & 0 & & \\
\hline & 0 & 1 & 2 & 3 & $\mathrm{k}$
\end{tabular}

or, symbolically,

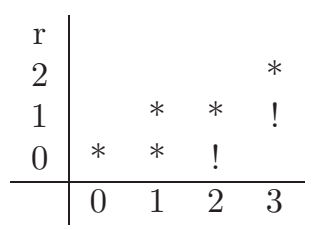


where we use "*" for integer points in $D_{1}$ and "!" for integer points in $D_{2}$.

There are two integer points in $D_{2}$, corresponding to $V_{1}^{1}(|a|)$ and $\operatorname{Hilb}_{(a, 2)}^{3} S$. The variety $V_{1}^{1}(|a|)$ classifies linear systems of type $g_{1}^{1}$ on smooth curves in $|a|$ and is evidently empty for any $(S, a)$. The variety $\operatorname{Hilb}_{(a, 2)}^{3} S$ classifies 0subschemes on $S$ of degree three with speciality index $\delta(\xi, a)=2$. Note that the linear system $|a|$ realizes $S$ as a quartic in $\mathbb{P}^{3}$ with at most double points. It follows that $\operatorname{Hilb}_{(a, 2)}^{3} S$ is empty when $\phi_{|a|}$ is an embedding, and parametrizes simple 0-cycles of the form $p+q+r$, where $p, q$ and $r$ are three distinct points on a rational curve contracted by $\phi_{|a|}$, and their degenerations. In particular, not that if Pic $S=\mathbb{Z}$, then $\operatorname{Hilb}_{(a, 2)}^{3} S$ is empty.

Now if Pic $S=\mathbb{Z}$, then the main theorem guarantees that all the varieties parametrized by points in $D_{3}$ are empty.

One can consider the set of integer triples $(r, d, k)$ which satisfy

$$
\operatorname{v} \cdot \operatorname{dim} B N_{k}(r, a, \beta) \geq 0
$$

For $d \leq 2 g-2$ this domain is shown on the Figure 10

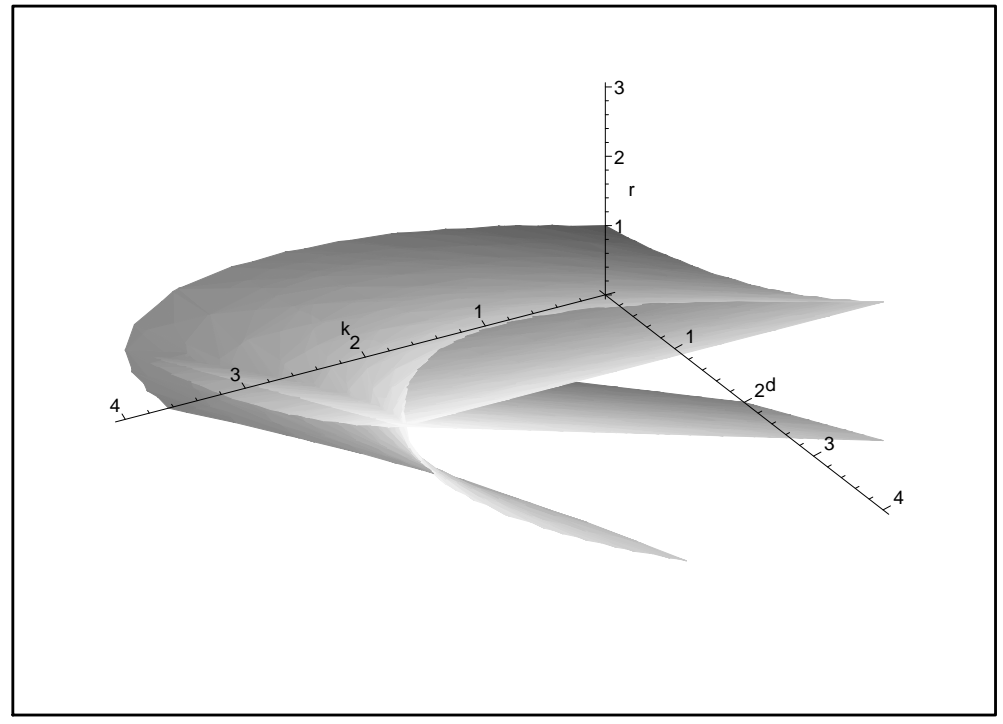

Figure 10: Geography for genus 3 


\subsection{Example: $g=4, d=5$}

Let $(S, a)$ be a polarized K3 surface of genus $g=4$, and let $d=5$. Note that $M(0, a, \beta)=M(0, a,-2) \subset \operatorname{Pic}^{1}(|a|)$. The formula 3.2 gives the following table of the Brill-Noether loci with nonnegative expected dimension,

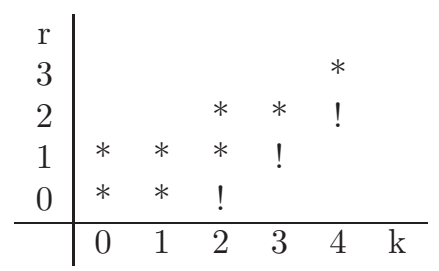

where the notations are the same as in the previous example.

There are three Brill-Noether schemes in the region $D_{2}$. For $r=0$ it is $V_{1}^{1}(|a|)$ which is empty. For $r=1$ it is $\operatorname{Hilb}_{(a, 3)}^{5} S$. A simple 0 -cycle $\xi \in \operatorname{Hilb}_{(a, 3)}^{5} S$ is an intersection of $S$ with a 3 -secant line $l$ in the projective embedding given by $|a|$. There is a net of hyperplanes in $\mathbb{P}^{4}$ through $l$, and each hyperplane cuts out a genus 4 curve on $S$ containing $\xi$. On such a curve $\xi$ gives a linear system of type $g_{5}^{3}$. Since a genus 5 curve does not have any $g_{5}^{3}$ 's, this variety is empty. (Essentially this proof, which is a reduction from the case $r=1$ to $r=0$, can be described as a correspondence between the Brill-Noether schemes $V_{2 g-2-d}^{r}\left(|a|^{s}\right)$ and $\left.\operatorname{Hilb}_{(r-\beta, a)}^{d}(S)\right)$.

If $r=2$ and Pic $S=\mathbb{Z} a$, then the emptiness of $B N_{4}(2, a,-2)$ follows from the discussion in 7.2

\subsection{Geometry of $W_{4}^{1}(C)$ for a trigonal curve of genus five}

(Compare 6]).

We will need the results of this example in the examples 8.6 and 8.5 For a slightly different approach, see [6].

Let $C$ be a trigonal curve of genus five. It is well known that $C$ has unique trigonal structure $g_{3}^{1}$. Let $t \in\left|g_{3}^{1}\right|$ be any divisor consisting of three distinct points. It follows that the linear span $\langle t\rangle$ is a trisecant line for $C$ in the canonical embedding of $C$. We denote this line as $l$.

Since $K_{C}-g_{3}^{1}=g_{5}^{2}, C$ has a (unique) structure of a plane quintic. Note that this linear system can be realized as a linear system of hyperplanes through $l$ in the canonical embedding of $C$. Let $C^{\prime}$ be the plane model of $C$. Since genus of a smooth plane quintic is equal to 6 , it follows that the of $C^{\prime}$ has one double point $q^{\prime}$, which can be either node or cusp.

Consider first the case when $q^{\prime}$ is a node, and let $q_{1}$ and $q_{2}$ be two preimages of $q^{\prime}$ on $C$. Since $K_{C}-g_{3}^{1}$ does not separate $q_{1}$ and $q_{2}$, we have $<l+q_{1}>=<l+q_{2}>$ for the linear spans of divisors. Let $\pi=<l+q_{i}>$; it is a two-dimensional plane in $\mathbb{P}^{4}$.

Note that the divisor $t+q_{1}+q_{2}$ is of type $g_{5}^{2}$. Let $H$ be a hyperplane in $\mathbb{P}^{4}$ through $\pi$. It intersects $C$ in a degree 3 divisor $t^{\prime}$ which forms $g_{3}^{1}$. Since $\left|K_{C}\right|=\left|t+t^{\prime}+q_{1}+q_{2}\right|$, It follows that $q_{1}+q_{2} \in\left|K_{C}-2 t\right|$. 
Let $p$ be any point on $C$. We define $L_{p}=g_{5}^{2}-p, M_{p}=g_{3}^{1}+p$. Note that the Serre duality interchanges $L_{p}$ and $M_{p}$ :

$$
K_{C} L_{p}^{-1} \simeq M_{p}
$$

We give the following geometric interpretation of $\left|L_{p}\right|$. Note that for any $p \in C$ we have $p \notin l$, since there are no linear systems of type $g_{4}^{2}$ on $C$. (Note that the definition of the linear span $\langle t+p\rangle$ includes an infinitesimal computation if $p \in t$.) It follows that $p i_{p}=\langle l+p\rangle$ is a two-plane in $\mathbb{P}^{4}$. Projection with center $\pi_{p}$ gives a linear system of type $g_{4}^{1}$ on $C$ which is equal to $\left|K_{C}-t-p\right|=$ $\left|g_{5}^{2}-p\right|=\left|L_{p}\right|$. (In terms of the plane model $C^{\prime},\left|L_{p}\right|$ is a projection from the point $p$ on $C^{\prime}$ ).

If $p \neq q_{i}$, then $\left|L_{p}\right|$ forms a $g_{4}^{1}$ without fixed points. Note that $q_{2}$ is a fixed point of $\left|L_{q_{1}}\right|$ and $q_{1}$ is a fixed point of $\left|L_{q_{2}}\right|$.

Note that

$$
\begin{gathered}
L_{q_{1}}=\left|K_{C}-t-q_{1}\right|=\left|t^{\prime}+q_{2}\right|=M_{q_{2}}, \\
L_{q_{2}}=M_{q_{1}}
\end{gathered}
$$

In particular, $K_{C} L_{q 1}^{-1}=M_{q_{1}}=L_{q_{2}}$.

Lemma. Any linear system of type $g_{4}^{1}$ on $C$ is of type $L_{p}$ or $M_{p}$ for some $p \in C$.

Proof. Let $L$ be a linear system of type $g_{4}^{1}$, and let $D \in|L|$ be a divisor consisting of 4 distinct points on $C: D=d_{1}+d_{2}+d_{3}+d_{4}$. Let $\pi=\langle D\rangle$; it is a 2 -plane in $\mathbb{P}^{4}$.

Let $t \in\left|g_{3}^{1}\right|$ be a divisor consisting of three distinct points, $t=t_{1}+t_{2}+t_{3}$. The linear span $l=\langle t\rangle$ is a trisecant line to $C$ in the canonical embedding.

Case 1: $l \in \pi$. Consider the set-theoretic union $E=D \cup t$. This is a set of 7, 6, 5 or 4 distinct points on $C$, and, correspondingly, $|E|$ is a linear system of type $g_{7}^{4}, g_{6}^{3}, g_{5}^{2}$ or $g_{4}^{1}$. Since $C$ does not have $g_{7}^{4}$ or $g_{6}^{3}$, the order of $E$ is either 4 or 5 .

If $|E|=g_{4}^{1}$, then we have $t \subset D$, i.e., $|D|=|t+p|=M_{p}$, and the lemma is proved. Let $|E|=g_{5}^{2}$. After re-numeration we have $D=t_{1}+t_{2}+d_{3}+d_{4}$. Note that the linear system $g_{5}^{2}=K_{C}-t$ does not separate $d_{3}$ and $d_{4}$, since $<t, d_{3}>=<t, d_{4}>=\pi$. It follows that, after renumeration, $d_{3}=q_{1}$ and $d_{4}=q_{2}$. In particular,

$$
|D|=\left|t_{1}+t_{2}+q_{1}+q_{2}\right|=\left|t-t_{3}+q_{1}+q_{2}\right|=\left|g_{5}^{2}-t_{3}\right|=L_{t_{3}},
$$

and the statement of the Lemma follows.

Case 2: $l \cap \pi=a$, where $a$ is a point in $\mathbb{P}^{4}$. Assume first that $a=t_{i}$ for some $i$, for example, $a=t_{1}$. Then the linear system $|D+t 1|$ is of type $g_{5}^{2}$ and is different from $K_{C}-t$, which gives a contradiction. It follows that $t_{i} \neq a$. The image of $\pi$ under the projection $p r_{l}: \mathbb{P}^{4} \rightarrow \mathbb{P}^{2}$ is a line in $\mathbb{P}^{2}$ containing four points of the image of $D$. Let $p$ be the fifth intersection point of this line with $C^{\prime}$. It follows that $|D|=\left|L_{p}\right|$, and the statement of the Lemma follows. 
Case 3: $l \cap \pi=\emptyset$. In this case one can repeat the construction of the case 2 , starting from the projection $p r_{l}$. E.o.p.

It follows that the variety $W=W_{4}^{1}(C)$ has two irreducible components, $W_{(1)}$ and $W_{(2)}$, each isomorphic to the curve $C$ itself: the isomorphism $C \rightarrow$ $W_{(1)}$ takes $p$ to $L_{p}$, and the isomorphism $C \rightarrow W_{(2)}$ takes $p$ to $M_{p}$. All the points on the first component, except from the images of $q_{1}$ and $q_{2}$, parametrize globally generated line bundles, while all the points on the second component parametrize linear systems with a base point. The Serre duality interchanges the two components (as well as points $q_{1}$ and $q_{2}$ ), as on the Figure [1]

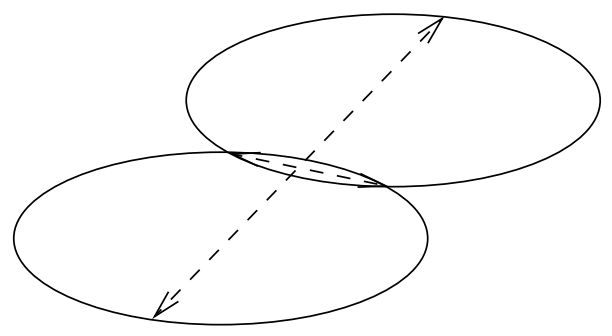

Figure 11: Geometry of $W_{4}^{1}(C)$.

In the case $C^{\prime}$ has a cusp $q^{\prime}$, the arguments of the previous case can be applied with $q_{1}=q_{2}$. Let $q$ be the preimage of $q^{\prime}$ on $C$. Then the variety $W_{4}^{1}(C)$ has two irreducible components, each isomorphic to $C$, touching each other at the point $q$.

\subsection{Example of a Brill-Noether special K3 of genus 5}

In this example we construct a polarized K3 surface $(S, a)$ of genus five such that every hyperplane section of $S$ is trigonal. We will use the existence of such a $\mathrm{K} 3$ in the examples 8.5 and 8.6 .

Let $F=F(0,1,2)$ be a 3 -dimensional scroll which can be constructed as a factor of the affine space $\mathbb{A}^{6}$ with coordinates $\left(t_{0}, t_{1}, x_{0}, x_{1}, x_{2}\right)$ by the action of the group $\mathbb{G}_{m} \times \mathbb{G}_{m}$, where the first factor acts with weights $(1,1,0,-1,-2)$ and the second one with weights $(0,0,1,1,1)$. The map $\pi: F \rightarrow \mathbb{P}^{1}$, where $\phi=t_{0} / t_{1}$, gives $F$ a structure of a two-dimensional projective bundle over $\mathbb{P}_{1}$.

Let $D$ be the subvariety in $F$ defined by the equation $x_{0}=0$. $D$ has a natural structure of a ruled surface isomorphic to the two-dimensional scroll $F(1,2)$. Let $M \in \operatorname{Pic} F$ be the image of $D$ in the Picard group of $F$, and let $L \in \operatorname{Pic} F$ be the class of a fiber of $\pi$. Let $\mathbb{P}=\mathbb{P}^{5}$. The map $\phi_{M}: F \rightarrow \mathbb{P}$ associated with the linear system $|M|$ can be written as $\phi_{M}=\left(x_{0}: t_{0} x_{1}: t_{1} x_{1}: t_{0}^{2} x_{2}: t_{0} t_{1} x_{2}: t_{1}^{2} x_{2}\right)$. Note that $M^{3}=3$ and $M^{2} L=1$ in the Chow ring of $F$.

Let $R$ be the subvariety in $F$ given by the equations $x_{1}=x_{2}=0 . \quad R$ is a smooth rational curve in $F$ disjoint from $D$ which is contracted to a point $p=(1,0,0,0,0,0) \in \mathbb{P}$ under the map $\phi_{M}$. One can check that $\left.\phi_{M}\right|_{F-R}$ is 
an embedding. It follows that $\phi_{M}(F)$ is a cone over a smooth scrollar surface $\phi_{M}(D)$ in $\mathbb{P}$. The geometry of the map $\phi_{M}$ is illustrated by the Figure 12

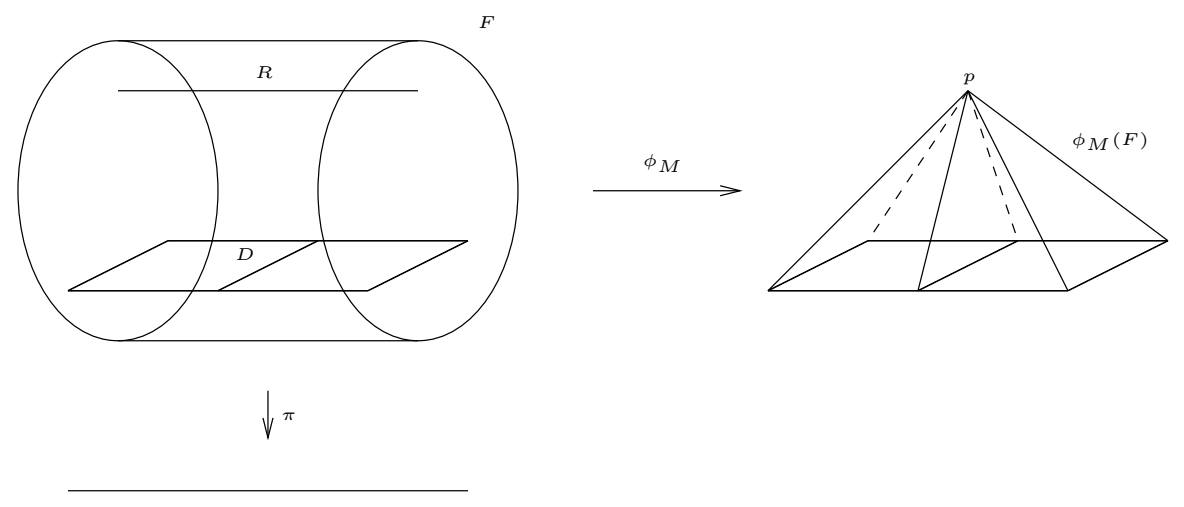

Figure 12: Construction of a "trigonal" K3, step 1

Let $H$ be the hyperplane in $\mathbb{P}$ defined by the vanishing of the first coordinate; we have $D=\phi_{M}(F) \cap H$.

One can check that $K_{F}=L-3 M$. Let $S$ be a smooth divisor in the linear system $\left|-K_{F}\right|=|-L+3 M|$ on $F$. For example, one can choose $S$ to be given by the "almost Weierstrass" equation

$$
x_{0}^{2} x_{1}=c_{5}(t) x_{2}^{3}+c_{3}(t) x_{2} x_{1}^{2}+c_{2}(t) x_{1}^{3},
$$

where $c_{k}(t)=c_{k}\left(t_{1}, t_{2}\right)$ is a generic homogeneous polynomials of degree $k$. Since $q_{S}=0$ and $K_{S}=0, S$ is a K3 surface.

Since

$$
R \cdot S=(M-L)(M-2 L)(3 M-L)=-1
$$

in the Chow ring of $F$, we have $R \subset S$. (It is also possible to see it directly, since the weight consideration implies that all the monomials in the equation of $S$ must be divisible by $x_{1}$ or by $x_{2}$.) Computing the Chern class gives $N_{S / R} \simeq$ $\mathcal{O}_{R}(-2)$, i.e., the map $\phi_{M}$ contracts the rational curve $R$ on $S$ into a double point $p \in \phi_{M}(S)$.

Let $C=S \cap D$. We get a diagram

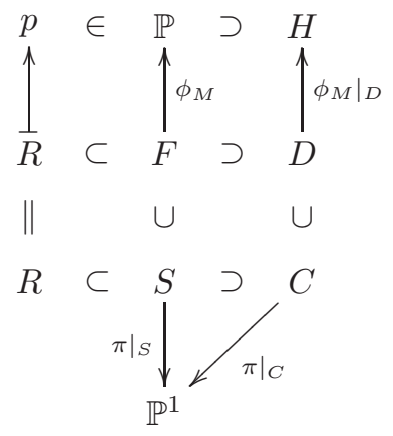


Note that

$$
\operatorname{deg}_{M} F=\operatorname{deg}_{M} D=M^{3}=3
$$

and

$$
\operatorname{deg}_{M} S=\operatorname{deg}_{M} C=M^{2}(3 M-L)=8
$$

One can see that $\left.\mathcal{O}_{F}(D)\right|_{C} \simeq K_{C}$. It follows that $\phi_{M}: C \rightarrow H \subset \mathbb{P}^{5}$ is the canonical embedding of $C$; in particular, $C$ has genus five.

The line bundle $\mathcal{O}_{F}(M)$, restricted to a fiber $F_{t}$, is isomorphic to $\mathcal{O}_{\mathbb{P}^{2}}(1)$, which implies that $\phi_{M}$ maps every rational curve $D_{t}$ to a trisecant line to $C$.

Fiber-wise with respect to $\pi$ the geometry of $S$ is shown on the Figure 13.

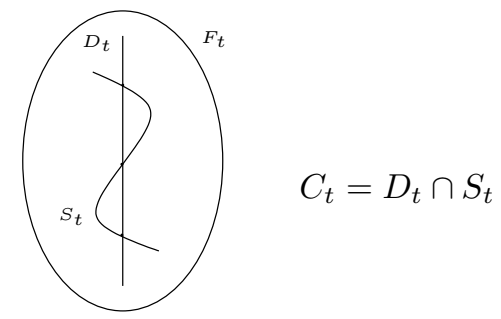

Figure 13: Construction of a "trigonal" K3, step 2

(Going in the opposite direction, consider the image of a non-hyperelliptic genus 5 curve $C$ under the canonical embedding $\phi_{K_{C}}: C \rightarrow \mathbb{P}^{4}$. The RiemannRoch theorem implies that there is a two-dimensional linear system of quadrics through $\phi_{K_{C}}(C)$ in $\mathbb{P}^{4}$. If $C$ is not trigonal, then $C$ is equal to the intersection of quadrics trough $C$ by Enriques-Petri theorem; if $C$ is trigonal, intersection of quadrics of through $C$ is a linear scrollar surface $X$ isomorphic to $F(1,2)$ such that every line on $X$ is trisecant to $C$.)

I learned about the geometry of scrollar varieties from Miles Reid's book "Chapters on Algebraic Surfaces."

We will use the existence of such a K3 surface in the next two examples.

8.5 Example: $g=5, d=5$

Let $(S, a)$ be a polarized K3 surface of genus $g=5$, and let $d=5$. Note that $M(0, a, \beta)=M(0, a,-1) \subset \operatorname{Pic}^{3}(|a|)$. The formula 3.2 gives the following geography for the Brill-Noether loci:

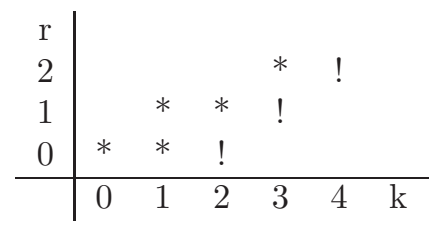


There are three integer points in the domain $D_{2}$. The variety $V_{3}^{1}(|a|)$ parametrizes trigonal structures on smooth curves in the linear system $|a|$. The variety $\operatorname{Hilb}_{(2, a)}^{5}$ parametrizes degree 50 -subschemes $\xi$ on $S$ which span a two-dimensional subspace in $\mathbb{P}^{5}$. For such a 0 -subscheme $\xi$ there is a net of hyperplane sections of $S$ through $\xi$, each smooth member $C$ of which is a genus five curve. On such a curve $C \xi$ gives a linear system of type $g_{5}^{2}$. (Note that the Serre dual to $g_{5}^{2}$ is $g_{3}^{1}$ for genus 5).

Taking into the account the results of [3], there are two cases to consider:

Case 1: There are no trigonal curves in the linear system $|a|^{s}$. In this case every smooth curve in $|a|$ is Brill-Noether general, and it follows that all the Brill-Noether varieties in the domain $D_{2}$ are empty. This is the case of a generic polarized K3 of genus 5. The results of Saint-Donat imply that such a K3 surface $S$ is an intersection of 3 quadrics in $\mathbb{P}^{5}$.

Case 2: All smooth curves in the linear system $|a|$ are trigonal. Since every smooth trigonal genus 5 curve has precisely one trigonal structure, the varieties $V_{3}^{1}(|a|)$ and $\operatorname{Hilb}_{(2, a)}^{5}(S)$ have dimension five, which is greater than one predicted by the formula [3.2).

Remark 1: results of Saint-Donat ( 3 ]) imply that such a K3 surface $S$ we have rank Pic $S \geq 2$, and Pic $S$ should contain the sublattice

$$
\left|\begin{array}{ll}
8 & 3 \\
3 & 0
\end{array}\right|
$$

(which is obviously true for the $\mathrm{K} 3$ constructed in the previous example). Note that Pic $S$ does not contain a nef divisor $H$ such that $(a, H)=1$. It follows that the main theorem of this text does not imply that the variety $B N_{4}(2, a,-1) \in$ $D_{2}$ is empty, since both of the the stability criteria we proved are not applicable in this case.

Remark 2: Let $(C, B) \in V_{3}^{1}(|a|)$. Note that both $B$ and $K_{C} B^{-1}$ are globally generated. By Lemma 5.1 there is a unique up to an isomorphism locally free globally generated extension $E$ of $(C, B)$ of rank 2. Since $\rho(C, B)<0$ (i.e., the generic genus 5 curve is not trigonal), the Riemann-Roch formula implies that $E$ is not simple and, in particular, is not stable.

Remark 3: Let $\xi \in \operatorname{Hilb}_{(2, a)}^{5}(S)$ be a simple 0 -cycle. We claim that $\xi$ is Caley-Bacharash with respect to $|a|$. First proof: $\xi$ induces a linear system of type $g_{5}^{2}$ on every curve $C \in|a|$ through $\xi$. Let $C^{\prime}$ be a plane model of $C$ given by this linear system. Then $\xi$ is the intersection of $C^{\prime}$ with a line $l$ on $\mathbb{P}^{2}$. Note that $K_{C}=\left.\mathcal{O}_{\mathbb{P}^{2}}(2 h)\right|_{C}$. Since every quadric on $\mathbb{P}^{2}$ through $\xi$ is of the form $l+l^{\prime}$, where $l^{\prime} \in|h|, \xi$ is Caley-Bacharash with respect to $|a|$. Second proof: if $\xi$ is not Caley-Bacharash, then it contains a sub-cycle $\xi^{\prime}$ of degree 4 and speciality index 2, which contradicts to the Martens-Mumford theorem applied to any curve through $\xi^{\prime}$.

It follows that the extension defined in the Lemma 5.1] is locally free. (We have already proved that it is not stable.) 
8.6 Example: $g=5, d=4$

Let $(S, a)$ be a polarized K3 surface of genus $g=5$, and let $d=4$. Note that $M(0, a, \beta)=M(0, a, 0) \subset \operatorname{Pic}^{4}(|a|)$. The formula 3.2 gives the following table of the expected dimensions of the Brill-Noether loci:

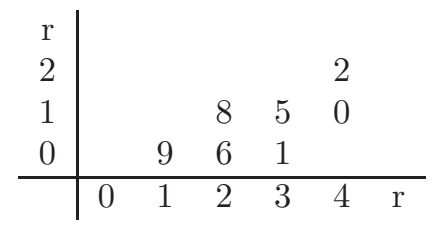

or, symbolically,

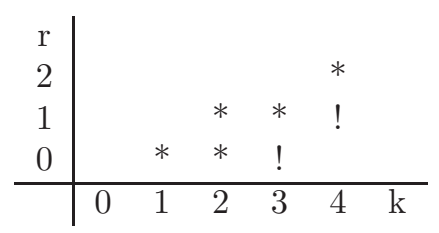

The variety $V_{4}^{2}\left(|a|^{s}\right)$ is empty by the Martens-Mumford theorem, and the geometry of $V_{4}^{1}\left(|a|^{s}\right)$ depends on the geometry of curves in the linear system $|a|$.

Case 1: There are no trigonal curves in $|a|^{s}$. If $C$ is a Brill-Noether general curve of genus 5 , it is easy to prove that $V(C):=V_{4}^{1}(C)$ is a smooth irreducible curve of genus 9. All linear systems parametrized by $V(C)$ are globally generated, and the Serre duality acts as an involution on $V(C)$. There are many more results on the geometry of $V(C)$ in [15].

Let $V(|a|)=V_{4}^{1}\left(|a|^{s}\right)$. This variety is fibered over $|a|^{s}$ with fibers $V_{4}^{1}(C)$.

The moduli space $S^{\prime}:=M_{H}(2, a, 0)$ is two-dimensional, and thus is a K3surface. There is a well-defined morphism $E: V(|a|) \rightarrow S^{\prime}$.

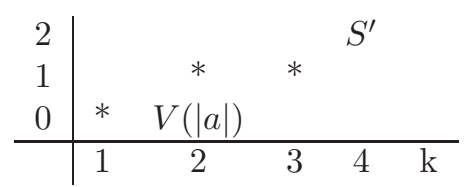

Case 2: All curves in the linear system $|a|^{s}$ are trigonal. The example of such a surface was constructed in the Example 8.4

Let $C$ be a trigonal curve of genus 5 . We proved in the Example 8.3 that $V(C):=W_{4}^{1}(C)$ is one-dimensional and has two irreducible components, $V_{(1)}(C)$ and $V_{(2)}(C)$, intersecting each other at two points or one double point. Generic points on the first component parametrize globally generated line bundles on $C$, and all points on the second component parametrize linear systems with base points. The Serre duality interchanges these two components. It follows that $V(|a|)=V_{4}^{1}\left(|a|^{s}\right)$ has two irreducible components, $V_{(1)}(|a|)$ and $V_{(2)}(|a|)$. Note that the generic point of $V_{(1)}(|a|)$ is not in the image of the correspondence $\mathcal{A}$.

Lemma: $S^{\prime}=M(2, a, 0)$ does not contain any globally generated vector bundles. 
Proof: Assume that $S^{\prime}$ contains a globally generated vector bundle $E$. Note that the Grassmanian variety $\operatorname{Gr}\left(2, h^{0}(S, E)\right)$ has positive dimension. Let us choose a generic point $V \in \operatorname{Gr}\left(2, h^{0}(S, E)\right)$. By lemma 5.3 the evaluation map $e_{(V, E)}$ has a good degeneration, and its cokernel is a point $v=(C, B) \in V_{4}^{1}\left(|a|^{s}\right)$ such that both $B$ and $K_{C} B^{-1}$ are globally generated. Since $V(|a|)$ does not contain such a point $p$, we get a contradiction.

Corollary Variety $\mathcal{A}_{2,0}^{4,2}$ is empty.

Remark. One can still define the extension morphism

$$
E: V(|a|) \rightarrow \mathrm{Coh}_{S}
$$

, where $\mathrm{Coh}_{S}$ is the stack of coherent sheaves on S. The stack Coh $\mathrm{C}_{S}$ has a substack $\mathrm{Coh}_{(1)}$ of non-locally free sheaves and a substack $\mathrm{Coh}_{(2)}$ of non-globally generated sheaves. Under $E, V_{(i)}(|a|)$ is the preimage of $\mathrm{Coh}_{(i)}$.

\subsection{Example of a Brill-Noether special K3 of genus 6}

In this example we construct a polarized K3-surface $(S, a)$ of genus six such that every hyperplane section of $S$ is isomorphic to a plane quintic. We will use the results of this Example in the Example 8.8

Let $(S, B)$ be a polarized genus K3 surface of genus two. Thus $B \in \operatorname{Pic}(S)$, $B^{2}=2$, and the map $\phi_{B}: S \rightarrow|B|^{\vee} \simeq \mathbb{P}^{2}$ realizes $S$ as a two-sheeted cover of $\mathbb{P}^{2}$ branched along a curve $R \subset \mathbb{P}^{2}$ of degree six. For a degree six curve $R$ given by the equation $f\left(x_{0}, x_{1}, x_{2}\right)=0$ the surface $S$ can be realized as a subscheme in the total space of the line bundle $\mathcal{O}_{\mathbb{P}^{2}}(3)$ given by the equation

$$
\zeta^{2}=f\left(x_{0}, x_{1}, x_{2}\right)
$$

Let $R$ be a plane sextic which has a tritangent line $l$, i.e., $f$, restricted to $l$, is a square of a homogeneous form of degree 3 . For example, we can take

$$
f=x_{1}^{6}+x_{2}^{6}+x_{0}^{5} x_{2}, l=\left(x_{2}=0\right),
$$

This is a very degenerate case, since all three points at which $l$ is bitangent to $R$ coincide. We have $\phi_{B}^{-1}(l)=\Gamma+\Gamma^{\prime}$, where $\Gamma$ and $\Gamma^{\prime}$ are smooth rational curves, and $(B, \Gamma)=1$.

Note that the linear system $a=2 B+\Gamma$ has genus 6 , and $(2 B+\Gamma, B)_{S}=5$. It follows that $|B|$, restricted to a curve in $|2 B+\Gamma|$, gives a linear system of type $g_{5}^{2}$. In particular, the image of $\phi_{2 B+\Gamma}: S \rightarrow|2 B+\Gamma|^{\vee} \simeq \mathbb{P}^{6}$ is as a projective K3 surface of genus such that all of its hyperplane sections are isomorphic to plane quintics (For the non-constructive approach, see [3]).

Note that since $(2 B+\Gamma, \Gamma)=0$, the map $\phi_{2 B+\Gamma}$ contracts $\Gamma$ into a point $p_{\Gamma}$.

Let $V_{1}=H^{0}(S, B)$ and $V_{2}=H^{0}(S, 2 B)$. Note that the canonical map

$$
\mathrm{Sym}^{2} V_{1} \rightarrow V_{2}
$$

is an isomorphism. Considering the adjunction sequences

$$
\left.0 \rightarrow \mathcal{O}(2 B) \rightarrow \mathcal{O}(2 B+\Gamma) \rightarrow \mathcal{O}(2 B+\Gamma)\right|_{\Gamma} \rightarrow 0
$$


and

$$
\left.0 \rightarrow \mathcal{O}(B) \rightarrow \mathcal{O}(2 B) \rightarrow \mathcal{O}(2 B)\right|_{B} \rightarrow 0
$$

on $S$, one can construct the diagram

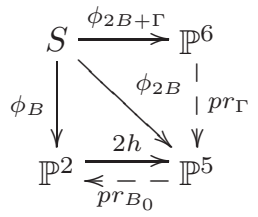

where $p r_{\Gamma}$ is a projection map with center $p_{\Gamma}$ and $p r_{B_{0}}$ is a projection from the (2-dimensional) linear span of any curve $B_{0} \in|B|$.

8.8 Example: $g=6, d=6$

Let $(S, a)$ be a polarized K3 surface of genus $g=6$, and let $d=6$. Note that $M(0, a, \beta)=M(0, a,-1) \subset \operatorname{Pic}^{4}(|a|)$. The formula 3.2 gives the following expected dimensions of the Brill-Noether loci:

\begin{tabular}{c|cccccccc}
$\mathrm{r}$ & & & & & & & \\
3 & & & & & & 0 & \\
2 & & & & 8 & 4 & & \\
1 & & 12 & 10 & 6 & 0 & & \\
0 & 12 & 10 & 6 & 0 & & & \\
\hline & 0 & 1 & 2 & 3 & 4 & 5 & $\mathrm{k}$
\end{tabular}

The Martens-Mumford theorem implies that $V_{4}^{2}(C)$ is empty for any genus 6 curve. It follows that $\operatorname{Hilb}_{3, a}^{6}(S)$ is empty. Let $V(|a|)=V_{4}^{1}(|a|)$ and $M=$ $M_{H}(3, a,-1)$. Symbolically, the geography of the Brill-Noether loci is

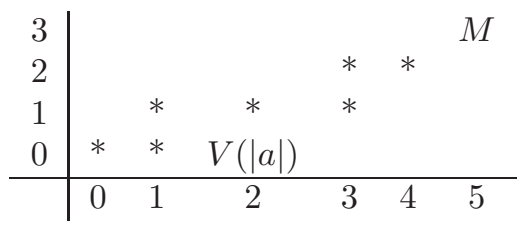

For a genus 6 curve one has $\rho\left(g_{4}^{1}\right)=0$ and $\operatorname{dim} V_{4}^{1}(C) \leq 1$ by the MartensMumford theorem. We will consider the following two cases:

Case 1: There is a smooth Brill-Noether general curve in the linear system $|a|$. Let $V(C)=V_{4}^{1}(C)$. It is well-known (cf. [6]) that for a Brill-Noether general curve $C$ of genus 6 the variety $V(C)$ is a union of 5 distinct points. One can easily check that every point in $V_{4}^{1}(C)$ parametrizes such a line bundle $L$ on $C$ that both $L$ and $K_{C} L^{-1}$ are globally generated.

The moduli space $M$ has dimension 0 . The results of [7] imply that $M$ is either empty or a reduced irreducible variety of dimension 0 (i.e., is a point). If Pic $S=\mathbb{Z} a$, there is a well-defined map $E: V(|a|) \rightarrow M$, which proves that $M$ 
is not empty, and since $E$ realizes $V(|a|)$ as a Grassmanian fibration over $M$, it follows that $V(|a|)$ is birational to the Grassmanian variety $\operatorname{Gr}(3,5)$ of dimension 6. Note that there exist a degree 5 morphism $V(|a|) \rightarrow|a|^{s} \subset|a| \simeq \mathbb{P}^{6}$.

Case 2: All curves in the linear system $|a|$ have $g_{5}^{2}$. An example of such a surface was constructed in 8.7

Let $C$ be a genus six curve which can be realized as a (smooth) plane quintic, and let $|h|$ be the corresponding linear system of type $g_{5}^{2}$. Projection map with center $p \in C$ gives a linear system of type $g_{4}^{1}$ on $C$, and one can prove that every $g_{4}^{1}$ on $C$ arises in such a way. It follows that $V_{4}^{1}(C) \simeq C$. Every linear system $B \in V_{4}^{1}(C)$ is globally generated, and every $K_{C}-B=2 h-(h-p)=h+p$ has a base point $p$. It follows that no $B \in V(C)$ have locally free extensions.

Vice versa, the only stable sheaf $E \in M$ is locally free by the ( [7), proposition 3.3 , but one can see as in the previous examples that $E$ is not globally generated.

It follows that the variety $\mathcal{A}_{3,0}^{5,3}$ is empty.

\section{References}

[1] Brill, A.; Noether, M, Uber die algebraischen Functionen und ihre Anwendugen in der Geometrie, Math. Ann. 7 (1873), 269-310.

[2] Grothendieck, Alexander, Technique de descente et theoremes d'existence en geometrie algebrique. V. Les schemas de Picard, Seminaire Bourbaki, Vol. 7, Exp. No. 232, 143-161.

[3] Saint-Donat, B., Projective models of K3 surfaces, Amer. J. Math. 96 (1974), 602-639.

[4] Mumford, David, Lectures on curves on an algebraic surface, Princeton University Press, Princeton, N.J. 1966

[5] Griffiths, P.; Harris, J., The dimension of the variety of special linear systems on a general curve, Duke Math. J. 47 (1980), 233-272

[6] Arbarello, E.; Cornalba, M.; Griffiths, P. A.; Harris, J., Geometry of algebraic curves. Vol. I., Springer-Verlag, New York, 1985

[7] Shigeru Mukai, On the moduli space of bundles on K3 surfaces, Tata Inst. Fund. Res., Bombay, 1987.

[8] Shigeru Mukai, Symplectic structure of the moduli space of sheaves on an abelian or K3 surface, Invent. Math. 77 (1984), no. 1, 101-116.

[9] Green, Mark; Lazarsfeld, Robert, A simple proof of Petri's theorem on canonical curves, Geometry today (Rome, 1984), 129-142.

[10] Lazarsfeld, Robert, Brill-Noether-Petri without degenerations, J. Differential Geom. 23 (1986), no. 3, 299-307. 
[11] Lazarsfeld, Robert, A sampling of vector bundle techniques in the study of linear series Lectures on Riemann surfaces (Trieste, 1987).

[12] Green, Mark; Lazarsfeld, Robert, Special divisors on curves on a K3 surface, Invent. Math. 89 (1987), no. 2, 357-370.

[13] Fulton, William, Intersection theory, Springer-Verlag, Berlin, 1984

[14] Tyurin, A. N., Cycles, curves and vector bundles on an algebraic surface, Duke Math. J. 54 (1987), no. 1, 1-26.

[15] Tyurin, A. N., On the intersection of quadrics , Uspekhi Matem. Nauk 30, v. 6 (186), (1975), 51-99.

[16] Griffiths, Phillip; Harris, Joseph, Principles of algebraic geometry, New York, 1994.

[17] Maruyama, Masaki, Construction of moduli spaces of stable sheaves via Simpson's idea, Lecture Notes in Pure and Appl. Math., 179.

[18] Le Potier, Joseph, Systemes coherents et structures de niveau, Asterisque No. 214 (1993)

[19] He, Min, Espaces de modules de syst'emes coherents, Internat. J. Math. 9 (1998), no. 5, 545.

[20] He, Min, Espaces de modules de syst'emes coherents. I. Normalit'e. C.R. Acad. Sci. Paris S'er. I Math. 325 (1997), no. 2, 183-188.

[21] He, Min, Espaces de modules de syst'emes coherents. II. Nombres de Donaldson. C.R. Acad. Sci. Paris S‘er. I Math. 325 (1997), no. 3, 301-306.

[22] Leyenson, Maxim, On the Brill-Noether theory for K3 surfaces, Part 2, in preparation. 